\title{
An exploratory study of host polymorphisms in genes that clinically characterize breast cancer tumors and pretreatment cognitive performance in breast cancer survivors
}

\author{
This article was published in the following Dove Press journal: \\ Breast Cancer - Targets and Therapy \\ 3 March 2017 \\ Number of times this article has been viewed
}

\author{
Theresa A Koleck ${ }^{1,2}$ \\ Catherine M Bender ${ }^{\prime}$ \\ Beth Z Clark ${ }^{3,4}$ \\ Christopher M Ryan ${ }^{5,6}$ \\ Puja Ghotkar' \\ Adam Brufsky ${ }^{4,7,8}$ \\ Priscilla F McAuliffe ${ }^{4,8,9}$ \\ Priya Rastogi ${ }^{4,7}$ \\ Susan M Sereika ${ }^{1,10,11}$ \\ Yvette P Conley ${ }^{1,12}$ \\ 'School of Nursing, University of \\ Pittsburgh, Pittsburgh, PA, ${ }^{2}$ School \\ of Nursing, Columbia University, \\ New York, NY, ${ }^{3}$ Division of \\ Gynecologic Pathology, Magee- \\ Womens Hospital of University of \\ Pittsburgh Medical Center (UPMC), \\ ${ }^{4}$ School of Medicine, ${ }^{5}$ Department of \\ Psychiatry, University of Pittsburgh, \\ Pittsburgh, PA, ${ }^{6}$ Department of \\ Psychiatry, University of California \\ San Francisco, San Francisco, CA, \\ ${ }^{7}$ Division of Hematology/Oncology, \\ Magee-Womens Hospital of UPMC, \\ ${ }^{8}$ University of Pittsburgh Cancer \\ Institute, ${ }^{9}$ Division of Breast Surgical \\ Oncology, Magee-Womens Hospital of \\ UPMC, ${ }^{10}$ Department of Biostatistics, \\ "Department of Epidemiology, \\ ${ }^{12}$ Department of Human Genetics, \\ Graduate School of Public Health, \\ University of Pittsburgh, Pittsburgh, \\ PA, USA
}

Correspondence: Theresa A Koleck School of Nursing, University of Pittsburgh, 3500 Victoria Street, Pittsburgh, PA I526I, USA

$\mathrm{Tel}+|\mathrm{I}| 2383764 \mid$

$\mathrm{Fax}+\mathrm{I} 4126248521$

Email tat30@pitt.edu
Purpose: Inspired by the hypothesis that heterogeneity in the biology of breast cancers at the cellular level may account for cognitive dysfunction symptom variability in survivors, the current study explored relationships between host single-nucleotide polymorphisms (SNPs) in 25 breast cancer-related candidate genes (AURKA, BAG1, BCL2, BIRC5, CCNB1, CD68, CENPA, CMC2, CTSL2, DIAPH3, ERBB2, ESR1, GRB7, GSTM1, MELK, MKI67, MMP11, MYBL2, NDC80, ORC6, PGR, RACGAP1, RFC4, RRM2, and SCUBE2), identified from clinically relevant prognostic multigene-expression profiles for breast cancer, and pretreatment cognitive performance. Patients and methods: The sample $(\mathrm{n}=220)$ was comprised of 138 postmenopausal women newly diagnosed with early stage breast cancer and 82 postmenopausal age- and educationmatched healthy controls without breast cancer. Cognitive performance was assessed after primary surgery but prior to initiation of adjuvant chemotherapy and/or hormonal therapy using a comprehensive battery of neuropsychological tests encompassing eight cognitive function composite domains: attention, concentration, executive function, mental flexibility, psychomotor speed, verbal memory, visual memory, and visual working memory. In total, 131 SNPs were included in the analysis. Standard and robust multiple linear regression modeling was used to examine relationships between each domain and the presence or absence of one or more minor alleles for each SNP. Genetic risk/protection scores (GRSs) were calculated for each domain to evaluate the collective effect of possession of multiple risk/protective alleles.

Results: With the exception of $C M C 2, M M P 11$, and $R A C G A P 1$, significant $(P<0.05)$ SNP main effect and/or SNP by future prescribed treatment group interactions were observed for every gene between at least one domain and one or more SNPs. All GRSs were found to be significantly $(P<0.001)$ associated with each respective domain score.

Conclusion: Associations between host SNPs and computed GRSs and variability in pretreatment cognitive function performance support the study hypothesis, and warrant further investigations to identify biomarkers for breast cancer-related cognitive dysfunction.

Keywords: breast neoplasms, genetics, cognition, biomarkers

\section{Introduction}

The recently published American Cancer Society/American Society of Clinical Oncology Breast Cancer Survivorship Care Guideline includes "assessment and management of physical and psychosocial long-term and late effects of breast cancer (BC) and treatment" as one of the five key areas of BC survivorship. ${ }^{1}$ Cognitive impairment related to cancer and cancer treatments is included in the guideline as a common and detrimental symptom experienced by BC survivors that can result in 
"distress and impaired [quality of life]". ${ }^{1}$ While assessment and management of cognitive dysfunction in BC survivors by clinicians are recommended, the guideline acknowledges that the causes of and treatment for cognitive dysfunction are not well established. ${ }^{1}$ The guideline does not include recommendations for clinicians on how to predict which survivors will experience cognitive difficulties, the severity of the difficulties, or the duration of the impairment either.

The lack of biomarkers available to enhance precision survivorship care is in stark contrast to those that have been developed to refine outcome prediction and selection of optimal therapy for BC. Specifically, the introduction of advanced genetic technologies into patient care has greatly enriched the cellular-level characterization of breast neoplasms and led to the development of clinically relevant prognostic multigene-expression profiles for BC. Briefly, prognostic multigene-expression profiles for $\mathrm{BC}$ use tumor gene-expression algorithm-driven estimation to enrich prediction of long-term $\mathrm{BC}$ outcomes, including recurrence or metastasis, and/or benefit of adjuvant therapies.

Considering that many investigators theorize that cognitive difficulties, especially prior to adjuvant chemotherapy and/or hormonal therapy, are related to the cancer itself,, ${ }^{2-5}$ we propose the use of BC-related genetic biomarkers for cognitive dysfunction symptom prediction and hypothesize that heterogeneity in BCs at the cellular level may account for variability in cognitive performance within the context of $\mathrm{BC}$. Because genes utilized in multigene-expression profiles for $\mathrm{BC}$ contribute to characterizing BCs at the cellular level in relation to aggressiveness and risk of progression, they represent ideal candidate genes for a biomarker study to test our hypothesis.

The potential use of BC-related genetic markers to account for cognitive difficulties among BC survivors is not without evidence. A growing number of studies are investigating associations between host genetic variability and alterations in cognitive performance in women diagnosed with and receiving treatment for $\mathrm{BC}$. Four published investigations have reported relationships between $A P O E$ and cognitive performance in women with BC. ${ }^{6-9}$ Associations with polymorphisms in genes involved in the dopamine and serotonin (ANKK1, BDNF, COMT, MTHFR, and SLC64A $)^{9-11}$ and DNA repair and oxidative stress (CAT, ERCC2, ERCC3, ERCC5, GPX1, PARP1, SEPP1, SOD1, and SOD2 $)^{12}$ pathways have also been reported. However, to the best of our knowledge, no previous investigations have focused on $\mathrm{BC}$-related genes as potential biomarkers for cognitive performance in women with BC.
To summarize, based on our hypothesis that heterogeneity in BCs at the cellular level may account for variability in cognitive performance within the context of $\mathrm{BC}$, this study was conducted to explore the contribution of host polymorphisms within candidate genes and their regulatory regions known to differentiate $\mathrm{BC}$ heterogeneity at the cellular level to pretreatment (ie, postsurgery, preadjuvant therapy) cognitive performance in postmenopausal women diagnosed with BC.

\section{Patients and methods Participants}

The sample $(\mathrm{n}=220)$ for this exploratory, genetic-association study was comprised of 138 postmenopausal women newly diagnosed with stage 1,2 , or $3 \mathrm{~A} \mathrm{BC}$ with no evidence of metastases and 82 postmenopausal age- and educationmatched healthy controls (HCs) without BC. Participants were initially enrolled in a study examining the effects of the adjuvant antiestrogen therapy, anastrozole \pm chemotherapy on cognitive function in postmenopausal women diagnosed with $\mathrm{BC}$ prior to, throughout, and following the antiestrogen-therapy regimen. ${ }^{13}$ Women diagnosed with $\mathrm{BC}$ were recruited from the Comprehensive BC Program of the University of Pittsburgh Cancer Institute. HC participants were obtained via referral from participants diagnosed with $\mathrm{BC}$, advertisements, and random-digit dialing through the University Center for Social and Urban Research. All study participants were 75 years of age or younger, able to speak and read English, and had completed a minimum of 8 years of education. Participants were excluded if they had a prior history of neurologic disease or cancer or had been hospitalized for psychiatric illness within the past 2 years. For this study, in order to account for the heterogeneity of $\mathrm{BC}$ tumors, women diagnosed with $\mathrm{BC}$ were further classified using prescribed future-treatment regimen as a surrogate for disease characteristics. Therefore, the analysis included two cohorts of women diagnosed with $\mathrm{BC}$ - those prescribed chemotherapy followed by anastrozole (prescribed $\mathrm{C}+\mathrm{A}$ ) $(\mathrm{n}=55)$ and those prescribed anastrozole only (prescribed AO) $(n=83)$ - as well as a cohort of HC women $(n=82)$. All participants provided written informed consent for study participation. Both the current genetic ancillary study and the parent study were approved by the University of Pittsburgh Institutional Review Board.

\section{Candidate-gene selection}

A total of 25 biologically plausible candidate genes that are theorized to characterize the biology of $\mathrm{BC}$ at the cellular 
level through utilization in prognostic multigene-expression profiles for $\mathrm{BC}$ were selected for investigation. Detailed rationale for selection and biological plausibility of candidate genes has been discussed previously. ${ }^{5}$ Prognostic multigene-expression profiles for $\mathrm{BC}$ use tumor geneexpression algorithm-driven estimation to enrich prediction of long-term cancer outcomes (ie, recurrence or metastasis) and/or benefit of adjuvant therapy. A number of multigeneexpression profiles for $\mathrm{BC}$ have been developed and include: the eleven-gene expression signature (Breast Cancer Index ${ }^{\mathrm{SM}}$; Biotheranostics, San Diego, CA, USA) ${ }^{14}$ the 14-gene prognostic expression signature (described in Tutt et al), ${ }^{15}$ the 21-gene BC assay (Oncotype DX ${ }^{\circledR}$ Breast Cancer Assay; Genomic Health, Redwood City, CA, USA), ${ }^{16,17}$ the 50-gene $\mathrm{BC}$ prognostic gene-signature assay (Prosigna ${ }^{\circledR}$ Breast Cancer Prognostic Gene Signature Assay; NanoString ${ }^{\circledR}$ Technologies Inc, Seattle, WA, USA) based on the PAM50 Breast Cancer Intrinsic Classifier, ${ }^{18}$ and the 70 -gene BC-recurrence assay (MammaPrint $^{\circledR}$ 70-gene Breast Cancer Recurrence Assay; Agendia ${ }^{\circledR}$, Irvine, CA, USA). ${ }^{19,20}$ While the profiles vary in the number of genes utilized, patient-eligibility criteria, and specific prognostic goal, genes included in these profiles play an important role in characterizing the biology of $\mathrm{BC}$ at the cellular level to address aggressiveness and risk of progression, and thus point to ideal candidates for an initial investigation of the study hypothesis.

A total of 21 of the 25 candidate genes (BAG1, BCL2, BIRC5, CCNB1, CENPA, CMC2, DIAPH3, ERBB2, ESR1, GRB7, MELK, MKI67, MMP11, MYBL2, NDC80, ORC6, $P G R, R A C G A P 1, R F C 4, R R M 2$, and SCUBE2) were prioritized for this investigation, based on duplication in two or more of the previously named multigene-expression profiles. ${ }^{12}$ Because the 21-gene $\mathrm{BC}$ assay is currently the most widely used profile in the US, the four remaining cancer genes used as part of this assay but not duplicated in another profile (AURKA, CD68, CTSL2, and GSTM1) were also prioritized.

\section{Single-nucleotide polymorphism (SNP) selection}

SNPs representing each candidate gene were selected. Functional or putatively functional (ie, known to influence expression levels, associated with $\mathrm{BC}$, or associated with a cognitive phenotype) polymorphisms within or directly upstream of candidate genes were identified from the literature. When a functional polymorphism was not identified and/or did not fully represent the gene of interest, tagging SNPs were selected using the Phase III HapMap database. Because the profiles from which candidate genes were selected rely upon gene-expression data, evaluation of DNA variability was extended $\pm 2,500$ bps beyond the gene to capture the UTR5' and UTR3' regulatory regions. Initial criteria for selection of tagging SNPs were as follows: $R^{2} \geq 0.8$, minor allele frequency (MAF) $\geq 0.2$, and selected for Caucasian ancestry, which represented the majority of study participants. The MAF criterion was ultimately relaxed to identify tagging SNPs for CTSL2, GRB7, MELK, MMP11, and RACGAP1. In addition, select polymorphisms in MIR125A, ${ }^{21,22} C C D C 170,{ }^{23-27}$ and $N F E 2 L 2^{28}$ were included to represent more fully ERBB2, $E S R 1$, and GSTM1, respectively. In total, 163 functional and tagging SNPs were identified.

\section{Genotype data collection and quality control}

Samples (3 $\mathrm{mL}$ of whole blood or $2 \mathrm{~mL}$ of saliva) were obtained for genotyping. DNA was extracted from peripheral blood leukocytes using a simple salting-out procedure ${ }^{29}$ or from saliva following the protocol and reagents supplied with Oragene ${ }^{\circledR}$ DNA-collection kits. ${ }^{30}$ The iPlex ${ }^{\circledR}$ MassArray platform (Sequenom, San Diego, CA, USA) was used as the primary genotyping method for this study. SNPs not conducive to genotyping with the iPlex platform were genotyped using TaqMan ${ }^{\circledR}$ allelic discrimination with the ABI Prism 7000 Sequence Detection System (SDS) and SDS software version 1.2.3 (Thermo Fisher Scientific, Waltham, MA, USA) or using a restriction fragment-length polymorphism-polymerase chain reaction approach.

Negative controls were included with all analyses. Genotypes were double-called by individuals blinded to participant phenotypes, and discrepancies were addressed by reviewing raw data or regenotyping. Participant genotypes were classified for data analysis based on the presence or absence of the minor allele (MA) (homozygous wild type compared to the combination of heterozygotes and homozygous-variant genotypes).

SNPs with call rates less than $90 \%$ or MAFs of less than 0.05 were omitted. For SNPs not meeting the $90 \%$ call-rate threshold but deemed essential for inclusion in the study (due to functional consequence, location within a candidate gene, or lack of alternative SNPs available within a given gene), secondary genotyping approaches were attempted. Alternative SNPs in linkage disequilibrium were selected for essential SNPs in instances of multiple failed genotyping attempts and/or lack of availability of alternative genotyping methods. Each SNP was tested for Hardy-Weinberg equilibrium (HWE) using $\chi^{2}$ goodness-of-fit or Fisher's exact tests to identify potential genotyping errors. 


\section{Pretreatment cognitive function evaluation}

Cognitive performance was assessed using a comprehensive battery of neuropsychological tests encompassing eight cognitive function composite domains:

- attention - Cambridge Neuropsychological Test Automated Battery (CANTAB) Rapid Visual Information Processing Test ${ }^{31}$

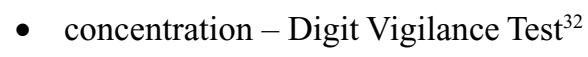

- executive function - CANTAB Stockings of Cambridge ${ }^{31}$ and CANTAB Spatial Working Memory ${ }^{31}$

- mental flexibility - Delis Kaplan Executive Function System Color-Word Interference Test ${ }^{33}$

- psychomotor speed - Grooved Pegboard $^{34}$ and Digit Symbol Substitution Test ${ }^{35}$

- verbal memory - Rey Auditory Verbal Learning Test, ${ }^{36}$ Verbal Fluency Test, and Rivermead Story Test ${ }^{37}$

- visual memory - CANTAB Paired Associates Learning ${ }^{31}$ and Rey Complex Figure Test ${ }^{38}$

- visual working memory - CANTAB Stockings of Cambridge $^{31}$ and Rey Complex Figure Test. ${ }^{38}$

Women with $\mathrm{BC}$ completed the battery after surgery, but before initiation of prescribed $\mathrm{C}+\mathrm{A}$ or $\mathrm{AO}$ adjuvant-therapy regimens. HCs completed the same neuropsychological test battery. Specifics related to the battery, creation of composite cognitive function domains, and $z$-score calculation have been reported previously. ${ }^{13}$ Please note that more negative $z$-scores designate poorer performance. Age (in years), estimated verbal intelligence (National Adult Reading Test - revised), ${ }^{39}$ depressive symptoms (Beck Depression Inventory II) ${ }^{40}$ anxiety (Profile of Mood States Tension-Anxiety subscale), ${ }^{41}$ fatigue (Profile of Mood States Fatigue-Inertia subscale), ${ }^{41}$ and current pain at time of assessment (Brief Pain Inventory $)^{42}$ were also recorded.

\section{Statistical analysis}

Stata version 14.1 (StataCorp, College Station, TX, USA) and Statistical Package for the Social Sciences (SPSS) versions 23 and 24 (IBM Corporation, Armonk, NY, USA) were used to perform statistical analyses. Descriptive statistics were computed. Standard and robust multiple linear regression modeling was used to examine relationships between each domain and the presence (ie, homozygous-variant genotype plus heterozygous genotype) or absence (ie, homozygous wild-type genotype) of one or more MAs for each SNP. Both main SNP effects only and SNP-prescribed treatment group-interaction effect-regression models were fitted. In all models, HCs served as the reference group for the two prescribed treatment groups (ie, prescribed $\mathrm{C}+\mathrm{A}$ or prescribed AO). Similarly, the wild-type genotype served as the reference group for possession of one or more MAs. All models were adjusted for age, estimated intelligence, and levels of depressive symptoms, anxiety, fatigue, and pain, and prescribed treatment group. Underlying assumptions were assessed. To lessen the impact of potentially influential points and adjust for heteroscedasticity, robust regression (generated using Huber weighting and biweighting iterations) model estimated regression coefficients and significance levels are reported.

Genetic risk/protection scores (GRSs) for each domain were then calculated to explore the influence of possession of multiple significant $(P<0.05)$ genotypes on domain scores, as previously described. ${ }^{12}$ SNP MAs that were significantly $(P<0.05)$ negatively or positively associated with a domain by either SNP main effects and/or SNP-prescribed treatment interaction effects were used in GRS calculations. A weighted calculation method, in which unstandardized robust regression coefficients from the individual models were multiplied by 0 (absence) or 1 (presence), based on a participant's genotype and prescribed treatment-group membership and then summed, was used to assign greater risk/protection to MAs with stronger associations. A lower GRS conveys greater genetic risk for poorer cognitive function, and a higher GRS conveys greater genetic protection. GRSs were added as the final predictor to standard and robust multiple linear regression models adjusted for age, estimated verbal intelligence, levels of depressive symptoms, anxiety, fatigue, pain, and prescribed treatment group. Only participants with all genetic data necessary for calculation of a GRS were included in the GRS analysis.

\section{Results}

\section{Participant characteristics}

A total of 220 participants $(n=55$ prescribed $C+A, n=83$ prescribed $\mathrm{AO}$, and $\mathrm{n}=82 \mathrm{HC}$ ) had genetic and complete covariate/confounder information and cognitive function scores available for one or more domains. A summary of overall demographic, covariate/confounder, and cognitive function data for participants included in this analysis can be found in Table 1.

Cohorts (ie, prescribed $\mathrm{C}+\mathrm{A}$, prescribed $\mathrm{AO}$, and $\mathrm{HC}$ ) differed statistically, yet not clinically meaningfully, by age and estimated verbal intelligence (Table 1). The groups also differed by level of anxiety $(P=0.003)$, with women with $\mathrm{BC}$ prescribed $\mathrm{C}+\mathrm{A}$ having higher mean pretreatment 
Table I Participant characteristics

\begin{tabular}{|c|c|c|c|c|c|c|c|}
\hline \multirow[t]{2}{*}{ Characteristics } & \multicolumn{3}{|l|}{ Total } & \multicolumn{4}{|l|}{ By cohort } \\
\hline & $\begin{array}{l}\text { Mean } \pm \text { SD } \\
\text { or } n(\%)\end{array}$ & Minimum & Maximum & $\begin{array}{l}\text { Prescribed } \\
C+A, n=55\end{array}$ & $\begin{array}{l}\text { Prescribed } \\
\text { AO, } n=83\end{array}$ & $\begin{array}{l}\text { Healthy } \\
\text { controls, } n=82\end{array}$ & $\begin{array}{l}\text { F-test }{ }^{\mathrm{a}} \text { or } \\
\chi^{2} / \text { Fisher's }^{\prime} \\
\text { exact test }^{\mathrm{b}}\end{array}$ \\
\hline Age (years) & $60.02 \pm 6.086$ & 43 & 75 & $58.76 \pm 5.467$ & $62.47 \pm 5.964$ & $58.39 \pm 5.858$ & $P<0.00 I^{*}$ \\
\hline Education (years) & $15.12 \pm 2.97$ & 9 & 29 & $15.67 \pm 2.783$ & $14.95 \pm 3.056$ & $14.93 \pm 2.993$ & $P=0.285$ \\
\hline $\begin{array}{l}\text { Estimated verbal intelligence } \\
\text { (NART-R) }\end{array}$ & $110.39 \pm 9.113$ & 82.42 & $|27.8|$ & $|08.94 \pm 8.87|$ & $107.04 \pm 8.844$ & II $4.74 \pm 7.796$ & $P<0.00 I^{*}$ \\
\hline Depressive symptoms (BDI-II) & $4.83 \pm 4.957$ & 0 & 29 & $5.24 \pm 4.615$ & $4.6 \pm 4.65$ & $4.79 \pm 5.495$ & $P=0.757$ \\
\hline $\begin{array}{l}\text { Anxiety (POMS Tension-Anxiety } \\
\text { subscale) }\end{array}$ & $7.47 \pm 5.536$ & 0 & 27 & $9.61 \pm 6.14$ & $6.97 \pm 4.654$ & $6.55 \pm 5.619$ & $P=0.003^{*}$ \\
\hline $\begin{array}{l}\text { Fatigue (POMS Fatigue-Inertia } \\
\text { subscale) }\end{array}$ & $5.61 \pm 5.942$ & 0 & 27 & $5.11 \pm 5.329$ & $5.84 \pm 6.352$ & $5.72 \pm 5.955$ & $P=0.763$ \\
\hline Pain (BPI pain right now) & $1.3 \pm 2.126$ & 0 & 9 & $1.47 \pm 1.96 \mathrm{I}$ & $1.55 \pm 2.265$ & $0.93 \pm 2.059$ & $P=0.13$ \\
\hline $\begin{array}{l}\text { Marital status (currently married or } \\
\text { living with significant other) }\end{array}$ & $139(63.2)$ & $\mathrm{N} / \mathrm{A}$ & $\mathrm{N} / \mathrm{A}$ & $38(69.1)$ & $54(65.1)$ & $47(57.3)$ & $P=0.348$ \\
\hline Number of children & $2 \pm 1.403$ & 0 & 8 & $1.75 \pm 1.22$ & $2.05 \pm 1.387$ & $2.13 \pm 1.522$ & $P=0.266$ \\
\hline Race (Caucasian) & $209(95)$ & $\mathrm{N} / \mathrm{A}$ & N/A & $52(94.5)$ & $81(97.6)$ & $76(92.7)$ & $P=0.305$ \\
\hline \multicolumn{8}{|l|}{ Cognitive function composite } \\
\hline \multicolumn{8}{|l|}{ Z-scores } \\
\hline Attention, $n=219$ & $-0.107 \pm 0.94939$ & -4.02 & 1.7 & $-0.052 \pm 0.937$ & $-0.202 \pm 1.017$ & $-0.047 \pm 0.889$ & $P=0.513$ \\
\hline Concentration, $n=219$ & $-0.056 \pm 0.8317$ & -2.2 & 2.5 & $-0.204 \pm 0.667$ & $-0.01 \pm 0.904$ & $-0.005 \pm 0.85$ & $P=0.322$ \\
\hline Executive function, $n=220$ & $-0.2357 \pm 0.64539$ & -1.69 & 2.41 & $-0.218 \pm 0.599$ & $-0.49 \pm 0.509$ & $0.01 \pm 0.705$ & $P<0.00 I^{*}$ \\
\hline Mental flexibility, $n=219$ & $0.0965 \pm 0.75203$ & -3.64 & 1.73 & $0.164 \pm 0.656$ & $0.09 \pm 0.786$ & $0.055 \pm 0.783$ & $P=0.707$ \\
\hline Psychomotor speed, $n=220$ & $-0.0548 \pm 0.88616$ & -3.67 & 1.22 & $0.07 I \pm 0.845$ & $-0.24 \pm 0.954$ & $0.048 \pm 0.819$ & $P=0.054$ \\
\hline Verbal memory, $\mathrm{n}=\mathbf{2 2 0}$ & $-0.1087 \pm 0.72263$ & -1.77 & 1.67 & $0.018 \pm 0.662$ & $-0.34 I \pm 0.638$ & $0.04 I \pm 0.786$ & $P=0.001 *$ \\
\hline Visual memory, $n=220$ & $0.0832 \pm 0.68602$ & -4.63 & 0.86 & $0.287 \pm 0.352$ & $0.009 \pm 0.708$ & $0.022 \pm 0.803$ & $P=0.038 *$ \\
\hline Visual working memory, $n=220$ & $0.0358 \pm 0.77624$ & -3.02 & 1.33 & $0.299 \pm 0.514$ & $-0.064 \pm 0.74 I$ & $-0.039 \pm 0.913$ & $P=0.014 *$ \\
\hline
\end{tabular}

Notes: $* P<0.05$. ${ }^{*}$ One-way ANOVAs utilized to compare mean values of continuous variables; 'Pearson's $\chi^{2}$ tests of independence, Fisher's exact test, or Fisher's exact test computed using two-sided Monte Carlo sampling based on 10,000 sampled tables used to examine associations between categorical variables. Only participants with complete confounder/covariate information were included in the participant-characteristic statistics.

Abbreviations: ANOVA, analyses of variance; AO, anastrozole only; BDI, Beck Depression Inventory; BPI, Brief Pain Inventory; C+A, chemotherapy plus anastrozole; NART-R, National Adult Reading Test - revised; POMS, Profile of Mood States; SD, standard deviation; N/A, not applicable.

anxiety levels $(9.61 \pm 6.14)$ than women with BC prescribed AO (6.97 \pm 4.654$)$ and HCs (6.55 \pm 5.619$)$. Comparison of tumor features by prescribed treatment group confirmed expected differences in disease characteristics (Table 2). To summarize, women with $\mathrm{BC}$ prescribed $\mathrm{C}+\mathrm{A}$ had higher frequencies of American Joint Committee on Cancer Stage $2 \mathrm{~A}, 2 \mathrm{~B}$, and $3 \mathrm{~A} \mathrm{BCs}$, larger mean tumor size, higher mean number of positive lymph nodes, higher mean Nottingham Score, greater frequency of lymphovascular invasion, lower ER H-score, greater frequency of HER2-positive cancer, higher mean Ki67 index, and higher mean Oncotype DX ${ }^{\circledR}$ BC Assay Recurrence Score ${ }^{\circledR}$ compared to women with BC prescribed AO.

No differences in covariates/confounders or pretreatment cognitive function $z$-scores were observed between HCs included in this ancillary genetic analysis and those enrolled in the parent study but not included in the genetic analysis $(\mathrm{n}=82)$. Women with $\mathrm{BC}$ prescribed $\mathrm{AO}$ included in the genetic analysis did have slightly lower $(P=0.044)$ mean estimated verbal intelligence $(107.04 \pm 8.844)$ than those enrolled in the parent study but not included in the genetic analysis $(n=155,109.42 \pm 8.542)$. Also, women with $\mathrm{BC}$ prescribed $\mathrm{C}+\mathrm{A}$ included in the genetic analysis had higher mean pretreatment verbal $(P=0.014,0.02 \pm 0.662)$, visual $(P=0.006,0.29 \pm 0.352)$, and visual working $(P=0.002$, $0.3 \pm 0.514)$ memory performance $z$-scores compared to those enrolled in the parent study but not included in the genetic analysis $(n=78 ;-0.28 \pm 0.697,0.03 \pm 0.615,-0.07 \pm 0.746$, respectively).

\section{Candidate-gene analysis}

Of the 163 SNPs originally identified, 32 SNPs that were not amenable to multiplexing, had call rates less than $90 \%$, or study MAFs of less than 0.05 were excluded. Alternatives were selected for three essential SNPs. In total, 131 SNPs were included in the genetic analysis (Table 3). Genotyping call rates for these SNPs ranged from $90 \%$ to $100 \%$. When considering all study participants, six SNPs were not 
Table 2 Tumor characteristics by study cohort

\begin{tabular}{|c|c|c|c|}
\hline Characteristics, mean \pm SD or $n(\%)$ & Prescribed $C+A, n=55$ & Prescribed $A O, n=83$ & F-test ${ }^{\mathrm{a}}$ or $\chi^{2} /$ Fisher's exact test ${ }^{\mathrm{b}}$ \\
\hline \multicolumn{4}{|l|}{ AJCC tumor stage, $n=130$} \\
\hline Stage I & $22(44)$ & $65(81.3)$ & $P<0.00 I^{*}$ \\
\hline Stage $2 A$ & $17(34)$ & $13(16.3)$ & \\
\hline Stage 2B & $6(12)$ & $2(2.5)$ & \\
\hline Stage 3A & $5(10)$ & 0 & \\
\hline Primary tumor size $(\mathrm{cm}), \mathrm{n}=\mathbf{I} 29$ & $2.16 \pm 1.484$ & $1.23 \pm 0.709$ & $P<0.00 I^{*}$ \\
\hline \multicolumn{4}{|l|}{ Lymph-node status, $n=\mid 29$} \\
\hline Positive & $19(38)$ & $5(6.3)$ & $P<0.00 I^{*}$ \\
\hline Negative & $31(62)$ & 74 (93.7) & \\
\hline Number of positive nodes, $n=130$ & $0.94 \pm 1.789$ & $0.06 \pm 0.244$ & $P<0.00 I^{*}$ \\
\hline \multicolumn{4}{|l|}{ Invasive type, $n=129$} \\
\hline Ductal & $45(90)$ & $63(79.7)$ & $P=0.323$ \\
\hline Lobular & $5(10)$ & $14(17.7)$ & \\
\hline Ductal and lobular & 0 & $2(2.5)$ & \\
\hline Nottingham score, $n=\mid 25$ & $6.60 \pm 1.370$ & $5.72 \pm 1.122$ & $P<0.00 I^{*}$ \\
\hline \multicolumn{4}{|l|}{ Nottingham grade, $n=\mid 25$} \\
\hline Grade I & $9(18)$ & $27(36)$ & $P<0.00 I^{*}$ \\
\hline Grade 2 & $26(52)$ & $44(58.7)$ & \\
\hline Grade 3 & $15(30)$ & $4(5.3)$ & \\
\hline \multicolumn{4}{|l|}{ ER status, $n=130$} \\
\hline Positive & $48(96)$ & $80(100)$ & $P=0.146$ \\
\hline Negative & $2(4)$ & 0 & \\
\hline ER H-score, $n=\mid 24$ & $240.08 \pm 73.684$ & $265.87 \pm 44.592$ & $P=0.017^{*}$ \\
\hline \multicolumn{4}{|l|}{ PR status, $n=130$} \\
\hline Positive & $38(76)$ & $7 \mid(88.8)$ & $P=0.055$ \\
\hline Negative & $12(24)$ & $9(I I .3)$ & \\
\hline PR H-score, $n=124$ & $110.35 \pm 101.612$ & $129.69 \pm 97.208$ & $P=0.289$ \\
\hline \multicolumn{4}{|l|}{ HER2 status, $n=125$} \\
\hline Positive & $9(19.1)$ & $4(5.1)$ & $P=0.017^{*}$ \\
\hline Negative & $38(80.9)$ & 74 (94.9) & \\
\hline \multicolumn{4}{|l|}{ LV invasion, $n=127$} \\
\hline Present & $21(42.9)$ & $6(7.7)$ & $P<0.00 I^{*}$ \\
\hline Absent & $28(57.1)$ & $72(92.3)$ & \\
\hline \multicolumn{4}{|l|}{ Ki67 classification, $n=68$} \\
\hline Low & $10(38.5)$ & I8 (42.9) & $P=0.114$ \\
\hline Moderate & $5(19.2)$ & $14(33.3)$ & \\
\hline High & $6(23.1)$ & $9(21.4)$ & \\
\hline Very high & $5(19.2)$ & I (2.4) & \\
\hline Ki67 index, $n=68$ & $28.73 \pm 26.834$ & $|7.3| \pm 13.337$ & $P=0.022^{*}$ \\
\hline Oncotype DX ${ }^{\circledR}$ Breast Cancer Assay & $26.52 \pm 9.774$ & $14.63 \pm 6.174$ & $P<0.00 I^{*}$ \\
\hline Recurrence Score $^{\circledR}, n=74$ & & & \\
\hline
\end{tabular}

Notes: ${ }^{*} P<0.05$. 'One-way ANOVAs utilized to compare mean values of continuous variables; 'Pearson's $\chi^{2}$ tests of independence, Fisher's exact test, or Fisher's exact test computed using two-sided Monte Carlo sampling based on 10,000 sampled tables used to examine associations between categorical variables. Only participants with complete confounder/covariate information were included in the participant-characteristic statistics.

Abbreviations: ANOVA, analyses of variance; AO, anastrozole only; C+A, chemotherapy plus anastrozole; HER2, human epidermal growth factor receptor 2; AJCC, American Joint Committee on Cancer; ER, estrogen receptor; PR, progesterone receptor; IHC, immunohistochemistry; LV, lymphovascular; SD, standard deviation.

in HWE: CTSL2 rs4361859 $(P=0.0078)$, ESR1 rs2234693 $(P=0.0344)$, ORC6 rs33994299 $(P=0.0051), P G R$ rs1042838 ( $P=0.0466), P G R$ rs $1042839(P=0.0103)$, and $P G R$ rs 474320 $(P=0.0434)$. In HC women alone, $P G R$ rs $1042838(P=0.016)$, $P G R$ rs $1042839(P=0.0027)$, and $P G R$ rs474320 $(P=0.0329)$ still did not meet HWE. We attributed the deviations from HWE to nonrandom sampling of study participants from the population leading to enrichment for BC in the cases and de-enrichment for $\mathrm{BC}$ in the controls for these genes known to be involved in $\mathrm{BC}$.

Individual polymorphisms significantly $(P<0.05)$ associated with a domain by either SNP main effects or SNP-prescribed treatment group-interaction effects are summarized by domain in Table 4. Overall, significant 
Table 3 SNPs included in genetic regression analyses $(n=220)$

\begin{tabular}{|c|c|c|c|c|}
\hline $\begin{array}{l}\text { Gene } \\
\text { SNP }\end{array}$ & $\begin{array}{l}\text { Wild-typel } \\
\text { variant } \\
\text { allele }^{\mathrm{a}}\end{array}$ & $\mathbf{n}$ & MAF & HWE $^{\mathrm{b}}$ \\
\hline \multicolumn{5}{|l|}{ AURKA } \\
\hline rsI047972 & $\mathrm{G} / \mathrm{A}$ & 219 & 0.148 & $P=I$ \\
\hline rs I6979877 & $\mathrm{A} / \mathrm{G}$ & 207 & 0.08 & $P=0.3725$ \\
\hline rs2273535 & $\mathrm{A} / \mathrm{T}$ & 213 & 0.216 & $P=0.9788$ \\
\hline rs6064389 & $\mathrm{G} / \mathrm{T}$ & 219 & 0.445 & $P=0.2279$ \\
\hline \multicolumn{5}{|l|}{ BAGI } \\
\hline rs706II8 & $\mathrm{T} / \mathrm{G}$ & 214 & 0.248 & $P=0.1553$ \\
\hline \multicolumn{5}{|l|}{ BCL2 } \\
\hline rsI564483 & $\mathrm{G} / \mathrm{A}$ & 207 & $0.27 \mid$ & $P=0.6855$ \\
\hline rs|7759659 & $\mathrm{A} / \mathrm{G}$ & 219 & 0.425 & $P=0.8885$ \\
\hline rs2279II5 & $\mathrm{A} / \mathrm{C}$ & 206 & 0.459 & $P=0.7047$ \\
\hline rs494II95 & $\mathrm{C} / \mathrm{A}$ & 218 & 0.429 & $P=0.423$ \\
\hline rs4987852 & $\mathrm{A} / \mathrm{G}$ & 218 & 0.078 & $P=1$ \\
\hline rs4987853 & $\mathrm{A} / \mathrm{G}$ & 217 & 0.189 & $P=0.5787$ \\
\hline rs4987855 & $\mathrm{G} / \mathrm{A}$ & 220 & $0.07 \mid$ & $P=0.6073$ \\
\hline rs956572 & $\mathrm{G} / \mathrm{A}$ & 211 & 0.398 & $P=0.6542$ \\
\hline rs9807663 & T/A & 218 & 0.108 & $P=0.7216$ \\
\hline \multicolumn{5}{|l|}{ BIRC5 } \\
\hline rs1042489 & $\mathrm{T} / \mathrm{C}$ & 217 & 0.359 & $P=0.548 \mathrm{I}$ \\
\hline rsI508I47 & $\mathrm{G} / \mathrm{A}$ & 218 & 0.358 & $P=0.5738$ \\
\hline rs|7878467 & $\mathrm{C} / \mathrm{T}$ & 217 & 0.111 & $P=1$ \\
\hline rs2239680 & $\mathrm{T} / \mathrm{C}$ & 214 & 0.299 & $P=0.7 \mid$ \\
\hline rs3764383 & $\mathrm{A} / \mathrm{G}$ & 213 & 0.305 & $P=0.9577$ \\
\hline rs8073069 & $\mathrm{G} / \mathrm{C}$ & 207 & 0.249 & $P=0.9445$ \\
\hline rs8073903 & $\mathrm{T} / \mathrm{C}$ & 212 & 0.366 & $P=0.6934$ \\
\hline rs990434I & $\mathrm{G} / \mathrm{C}$ & 206 & 0.318 & $P=0.706 \mathrm{I}$ \\
\hline \multicolumn{5}{|l|}{ CCNBI } \\
\hline rs 164390 & $\mathrm{G} / \mathrm{T}$ & 214 & $0.37 \mid$ & $P=0.52$ \\
\hline rs350099 & $\mathrm{T} / \mathrm{C}$ & 216 & 0.396 & $P=0.5396$ \\
\hline rs350I04 & $\mathrm{T} / \mathrm{C}$ & 219 & 0.459 & $P=0.564$ \\
\hline \multicolumn{5}{|l|}{ CD68 } \\
\hline rs8066665 & $\mathrm{G} / \mathrm{A}$ & 220 & 0.457 & $P=0.1667$ \\
\hline rs9901673 & $\mathrm{C} / \mathrm{A}$ & 218 & 0.172 & $P=0.0915$ \\
\hline \multicolumn{5}{|l|}{ CENPA } \\
\hline rs38065I7 & $\mathrm{A} / \mathrm{G}$ & 215 & 0.34 & $P=0.8 \mathrm{I} I \mathrm{I}$ \\
\hline rs38065I8 & $\mathrm{T} / \mathrm{C}$ & 214 & 0.278 & $P=0.8532$ \\
\hline \multicolumn{5}{|l|}{ CMC2 } \\
\hline rs1025065 & $\mathrm{C} / \mathrm{A}$ & 209 & 0.361 & $P=0.8277$ \\
\hline rs1981867 & $\mathrm{C} / \mathrm{T}$ & 220 & 0.307 & $P=0.1739$ \\
\hline rs9936489 & $\mathrm{T} / \mathrm{G}$ & 215 & 0.319 & $P=0.7119$ \\
\hline \multicolumn{5}{|l|}{ CTSL2 } \\
\hline rsl6919034 & $\mathrm{A} / \mathrm{G}$ & 213 & 0.169 & $P=0.350 \mathrm{I}$ \\
\hline rs4361859 & $\mathrm{A} / \mathrm{G}$ & 219 & 0.327 & $\begin{array}{l}P=0.0078^{*} ; \\
P=0.0695^{\mathrm{HC}}\end{array}$ \\
\hline \multicolumn{5}{|l|}{ DIAPH3 } \\
\hline rs|337652 & $\mathrm{G} / \mathrm{A}$ & 217 & 0.212 & $P=0.9194$ \\
\hline rs4547237 & $\mathrm{A} / \mathrm{G}$ & 220 & 0.307 & $P=0.8219$ \\
\hline \multicolumn{5}{|l|}{ ERBB2 } \\
\hline rsl058808 & $\mathrm{G} / \mathrm{C}$ & 217 & 0.373 & $P=0.2746$ \\
\hline rsII3620I & $\mathrm{A} / \mathrm{G}$ & 220 & 0.232 & $P=0.9465$ \\
\hline rs|476278 & $\mathrm{A} / \mathrm{G}$ & 220 & 0.357 & $P=0.9976$ \\
\hline rs1810132 & $\mathrm{T} / \mathrm{C}$ & 212 & 0.318 & $P=0.1537$ \\
\hline rs25I7955 & $\mathrm{T} / \mathrm{C}$ & 220 & 0.373 & $P=0.4823$ \\
\hline
\end{tabular}

Table 3 (Continued)

\begin{tabular}{|c|c|c|c|c|}
\hline $\begin{array}{l}\text { Gene } \\
\text { SNP }\end{array}$ & $\begin{array}{l}\text { Wild-type/ } \\
\text { variant } \\
\text { allele }^{\mathrm{a}}\end{array}$ & $\mathbf{n}$ & MAF & HWE $^{\mathrm{b}}$ \\
\hline rs4252596 & $\mathrm{C} / \mathrm{A}$ & 220 & 0.121 & $P=0.7482$ \\
\hline rs90350I & $\mathrm{G} / \mathrm{A}$ & 211 & 0.332 & $P=0.3885$ \\
\hline rs9303274 & $\mathrm{C} / \mathrm{T}$ & 219 & 0.356 & $P=0.9485$ \\
\hline rsI2976445(MIR/25A) & $\mathrm{T} / \mathrm{C}$ & 220 & 0.298 & $P=0.1467$ \\
\hline \multicolumn{5}{|l|}{ ESRI } \\
\hline rs10484919 & $\mathrm{C} / \mathrm{T}$ & 204 & 0.088 & $P=0.6574$ \\
\hline rs 1062577 & T/A & 215 & $0.08 \mathrm{I}$ & $P=0.1495$ \\
\hline rsII96428I & $\mathrm{C} / \mathrm{T}$ & 214 & 0.075 & $P=1$ \\
\hline rs 12173570 & $\mathrm{C} / \mathrm{T}$ & 219 & 0.132 & $P=0.3857$ \\
\hline rs 12665044 & $\mathrm{C} / \mathrm{T}$ & 213 & 0.132 & $P=0.7665$ \\
\hline rsI5I4348 & $\mathrm{A} / \mathrm{C}$ & 220 & 0.468 & $P=0.9519$ \\
\hline rs 1801132 & $\mathrm{C} / \mathrm{G}$ & 220 & 0.232 & $P=0.4098$ \\
\hline rsl88405I & A/G & 207 & 0.336 & $P=0.917$ \\
\hline rs2046210 & $\mathrm{C} / \mathrm{T}$ & 213 & 0.357 & $P=0.352$ \\
\hline rs207|454 & $\mathrm{T} / \mathrm{G}$ & 213 & 0.11 & $P=I$ \\
\hline rs2077647 & $\mathrm{G} / \mathrm{A}$ & 217 & 0.484 & $P=0.0643$ \\
\hline rs2228480 & $\mathrm{G} / \mathrm{A}$ & 218 & 0.188 & $P=0.2292$ \\
\hline rs2234693 & $\mathrm{C} / \mathrm{T}$ & 206 & $0.48 I$ & $\begin{array}{l}P=0.0344^{*} ; \\
P=0.9104^{\mathrm{HC}}\end{array}$ \\
\hline rs2347867 & $\mathrm{A} / \mathrm{G}$ & 215 & 0.34 & $P=0.5008$ \\
\hline rs2744677 & $\mathrm{A} / \mathrm{C}$ & 215 & 0.249 & $P=0.6319$ \\
\hline rs28I3543 & $\mathrm{G} / \mathrm{A}$ & 213 & 0.181 & $P=0.9848$ \\
\hline rs28I3544 & $\mathrm{A} / \mathrm{G}$ & 215 & 0.235 & $P=0.05 / 2$ \\
\hline rs294I740 & $\mathrm{T} / \mathrm{C}$ & 220 & 0.391 & $P=0.4585$ \\
\hline rs3020314 & $\mathrm{T} / \mathrm{C}$ & 208 & 0.358 & $P=0.4848$ \\
\hline rs3778099 & $\mathrm{T} / \mathrm{C}$ & 208 & 0.089 & $P=1$ \\
\hline rs3798577 & $\mathrm{T} / \mathrm{C}$ & 220 & 0.475 & $P=0.277$ \\
\hline rs488I33 & $\mathrm{C} / \mathrm{T}$ & 209 & 0.285 & $P=0.5 \mathrm{I} 0 \mathrm{I}$ \\
\hline rs5320I0 & $\mathrm{T} / \mathrm{C}$ & 212 & 0.434 & $P=0.7636$ \\
\hline rs6557|7I & $\mathrm{C} / \mathrm{T}$ & 218 & 0.303 & $P=0.7528$ \\
\hline rs77275268 & $\mathrm{C} / \mathrm{T}$ & 217 & 0.083 & $P=0.1827$ \\
\hline rs776II33 & $\mathrm{T} / \mathrm{C}$ & 216 & 0.153 & $P=0.584$ \\
\hline rs7761846 & $\mathrm{T} / \mathrm{C}$ & 204 & 0.054 & $P=0.450 \mathrm{I}$ \\
\hline rs7766585 & $\mathrm{T} / \mathrm{G}$ & 218 & 0.154 & $P=0.1375$ \\
\hline rs7767|43 & $A / G$ & 214 & 0.243 & $P=0.0849$ \\
\hline rs82742I & $\mathrm{C} / \mathrm{T}$ & 212 & 0.467 & $P=0.1489$ \\
\hline rs851967 & $\mathrm{G} / \mathrm{A}$ & 216 & 0.308 & $P=0.638$ \\
\hline rs85I97I & $\mathrm{G} / \mathrm{A}$ & 216 & 0.313 & $P=0.5073$ \\
\hline rs85।982 & $\mathrm{T} / \mathrm{C}$ & 217 & 0.362 & $P=0.3 \mid 78$ \\
\hline rs85। 998 & $\mathrm{C} / \mathrm{T}$ & 219 & 0.313 & $P=0.654$ \\
\hline rs910416 & $\mathrm{T} / \mathrm{C}$ & 220 & 0.491 & $P=0.996 \mathrm{I}$ \\
\hline rs9322331 & $\mathrm{C} / \mathrm{T}$ & 215 & 0.381 & $P=0.8339$ \\
\hline rs9340799 & $\mathrm{A} / \mathrm{G}$ & 213 & 0.397 & $P=0.478 I$ \\
\hline rs9383938 & $\mathrm{G} / \mathrm{T}$ & 218 & 0.083 & $P=0.372 I$ \\
\hline rs9397435 & $\mathrm{A} / \mathrm{G}$ & 220 & 0.073 & $P=0.6108$ \\
\hline rs9397456 & $\mathrm{G} / \mathrm{A}$ & 203 & 0.217 & $P=0.1522$ \\
\hline rs985694 & $\mathrm{C} / \mathrm{T}$ & 218 & 0.12 & $P=0.773 \mid$ \\
\hline rsl038304(CCDCI 70) & $\mathrm{G} / \mathrm{A}$ & 218 & 0.456 & $P=0.1394$ \\
\hline $\mathrm{rs} / 2662670(C C D C I 70)$ & $\mathrm{T} / \mathrm{G}$ & 217 & 0.069 & $P=0.274$ \\
\hline rs3734805(CCDCI 70) & $\mathrm{A} / \mathrm{C}$ & 213 & 0.075 & $P=0.2359$ \\
\hline rs37573I8(CCDCI 70$)$ & $\mathrm{G} / \mathrm{A}$ & 213 & 0.059 & $P=0.3629$ \\
\hline rs6929|37(CCDCI 70) & $\mathrm{G} / \mathrm{A}$ & 216 & 0.319 & $P=0.34 \mathrm{I} I$ \\
\hline
\end{tabular}


Table 3 (Continued)

\begin{tabular}{|c|c|c|c|c|}
\hline $\begin{array}{l}\text { Gene } \\
\text { SNP }\end{array}$ & $\begin{array}{l}\text { Wild-typel } \\
\text { variant } \\
\text { allele }^{\mathrm{a}}\end{array}$ & $\mathbf{n}$ & MAF & HWE $^{\mathrm{b}}$ \\
\hline \multicolumn{5}{|l|}{ GRB7 } \\
\hline rs9910678 & $\mathrm{T} / \mathrm{C}$ & 218 & 0.053 & $P=0.1079$ \\
\hline \multicolumn{5}{|l|}{ GSTMI } \\
\hline rsI0654II & $\mathrm{C} / \mathrm{G}$ & 209 & 0.194 & $P=0.0884$ \\
\hline rs4I 2543 & $\mathrm{G} / \mathrm{C}$ & 216 & 0.081 & $P=0.3714$ \\
\hline rs35652 I24(NFE2L2) & $\mathrm{T} / \mathrm{C}$ & 218 & 0.298 & $P=0.2739$ \\
\hline rs672I96I(NFE2L2) & $\mathrm{G} / \mathrm{T}$ & 212 & 0.101 & $P=1$ \\
\hline \multicolumn{5}{|l|}{ MELK } \\
\hline rs10973007 & $\mathrm{C} / \mathrm{G}$ & 209 & 0.189 & $P=0.8337$ \\
\hline rs 2250340 & $\mathrm{C} / \mathrm{T}$ & 220 & 0.075 & $P=1$ \\
\hline rs3780350 & $\mathrm{C} / \mathrm{T}$ & 213 & 0.155 & $P=0.6424$ \\
\hline \multicolumn{5}{|l|}{ MKI67 } \\
\hline rs 10732438 & $\mathrm{~A} / \mathrm{G}$ & 211 & 0.367 & $P=0.1859$ \\
\hline rs1076475। & $\mathrm{A} / \mathrm{C}$ & 220 & 0.239 & $P=0.5706$ \\
\hline \multicolumn{5}{|l|}{ MMPII } \\
\hline rsl3|45I & $\mathrm{T} / \mathrm{C}$ & 216 & 0.107 & $P=1$ \\
\hline \multicolumn{5}{|l|}{ MYBL2 } \\
\hline rsII556379 & $\mathrm{C} / \mathrm{G}$ & 220 & 0.05 & $P=0.4243$ \\
\hline rs2070235 & $\mathrm{A} / \mathrm{G}$ & 220 & 0.093 & $P=1$ \\
\hline rs619289 & $\mathrm{C} / \mathrm{T}$ & 216 & 0.197 & $P=0.7837$ \\
\hline rs826943 & $\mathrm{T} / \mathrm{C}$ & 213 & 0.146 & $P=0.7823$ \\
\hline rs826944 & $\mathrm{C} / \mathrm{T}$ & 219 & 0.142 & $P=1$ \\
\hline \multicolumn{5}{|l|}{ NDC80 } \\
\hline rs 12408485 & $\mathrm{~A} / \mathrm{G}$ & 203 & 0.382 & $P=0.473 \mid$ \\
\hline rs2292274 & $\mathrm{T} / \mathrm{C}$ & 207 & 0.268 & $P=0.5054$ \\
\hline \multicolumn{5}{|l|}{ ORC6 } \\
\hline rs33994299 & $\mathrm{T} / \mathrm{C}$ & 220 & 0.475 & $\begin{array}{l}P=0.005 I^{*} ; \\
P=0.1405^{\mathrm{HC}}\end{array}$ \\
\hline \multicolumn{5}{|l|}{ PGR } \\
\hline rsl042838 & $\mathrm{G} / \mathrm{T}$ & 216 & 0.141 & $\begin{array}{l}P=0.0466^{*} ; \\
P=0.016^{\mathrm{HC}, *}\end{array}$ \\
\hline rsI042839 & $\mathrm{C} / \mathrm{T}$ & 208 & 0.13 & $\begin{array}{l}P=0.0103^{*} ; \\
P=0.0027^{H C}, *\end{array}$ \\
\hline rs 10895068 & $\mathrm{G} / \mathrm{A}$ & 214 & 0.063 & $P=1$ \\
\hline rsII22456I & $\mathrm{C} / \mathrm{T}$ & 214 & 0.119 & $P=0.746$ \\
\hline rsl893505 & $\mathrm{C} / \mathrm{T}$ & 220 & 0.382 & $P=0.7593$ \\
\hline rs 1942836 & $\mathrm{~T} / \mathrm{C}$ & 217 & 0.201 & $P=0.7604$ \\
\hline rs47I767 & $\mathrm{A} / \mathrm{G}$ & 216 & 0.313 & $P=0.3574$ \\
\hline rs 474320 & $\mathrm{~T} / \mathrm{A}$ & 197 & 0.147 & $\begin{array}{l}P=0.0434^{*} ; \\
P=0.0329^{H C}, *\end{array}$ \\
\hline rs4754732 & $\mathrm{T} / \mathrm{C}$ & 220 & 0.334 & $P=0.178$ \\
\hline rs484389 & $\mathrm{T} / \mathrm{C}$ & 212 & 0.217 & $P=0.6804$ \\
\hline rs568I57 & $A / G$ & 219 & 0.493 & $P=0.312$ \\
\hline rs590688 & $\mathrm{C} / \mathrm{G}$ & 215 & 0.463 & $P=0.1662$ \\
\hline rs608995 & $\mathrm{A} / \mathrm{T}$ & 218 & 0.22 & $P=0.5724$ \\
\hline \multicolumn{5}{|l|}{ RACGAPI } \\
\hline rs730353I & $\mathrm{G} / \mathrm{A}$ & 214 & 0.058 & $P=1$ \\
\hline \multicolumn{5}{|l|}{ RFC4 } \\
\hline rs|35409| & $\mathrm{A} / \mathrm{C}$ & 214 & 0.238 & $P=0.9537$ \\
\hline \multicolumn{5}{|l|}{ RRM2 } \\
\hline rsII38729 & $\mathrm{A} / \mathrm{G}$ & 202 & 0.136 & $P=0.382 \mathrm{I}$ \\
\hline rs430955I & $\mathrm{C} / \mathrm{T}$ & 218 & 0.452 & $P=0.8925$ \\
\hline rs4668664 & $\mathrm{G} / \mathrm{A}$ & 215 & 0.263 & $P=0.685$ \\
\hline
\end{tabular}

(Continued)
Table 3 (Continued)

\begin{tabular}{lllll}
\hline $\begin{array}{l}\text { Gene } \\
\text { SNP }\end{array}$ & $\begin{array}{l}\text { Wild-typel } \\
\text { variant } \\
\text { allele }^{\mathbf{a}}\end{array}$ & $\mathbf{n}$ & MAF & HWE $^{\mathrm{b}}$ \\
\hline SCUBE2 & T/G & 213 & $0.21 \mathrm{I}$ & $P=0.8348$ \\
$\mathrm{rs} \mid 136966$ & $\mathrm{C} / \mathrm{T}$ & 219 & 0.47 & $P=0.4879$ \\
$\mathrm{rs} 4910440$ & $\mathrm{~A} / \mathrm{G}$ & 207 & 0.266 & $P=0.198$ \\
$\mathrm{rs} 6486125$ & &
\end{tabular}

Notes: ${ }^{*} P<0.05$. ${ }^{a}$ Wild-type and variant alleles based on study sample; ${ }^{b} \chi^{2}$ goodnessof-fit or exact-test $P$-value.

Abbreviations: $\mathrm{HC}$, healthy control; HWE, Hardy-Weinberg equilibrium; MAF, minor allele frequency; SNP, single-nucleotide polymorphism.

relationships were noted between at least one domain and one or more polymorphisms of all candidate genes, except CMC2, MMP11, and RACGAP1. Comprehensive results from the individual SNP and cognitive function regression analyses are located in Table S1.

Therefore, one or more polymorphisms from the following genes, through either main SNP effects or SNPprescribed treatment group-interaction effects, were included in GRSs: attention - ERBB2-MIR125A, ESR1, $M Y B L 2$, and $S C U B E 2$; concentration - AURKA, BCL2, CCNB1, CENPA, DIAPH3, ESR1, ESR1-CCDC170, GRB7, $M E L K$, and $P G R$; executive function - BAG1, BCL2, CCNB1, CTSL2, DIAPH3, ESR1, GSTM1, MELK, MYBL2, $P G R$, and SCUBE2; mental flexibility - BCL2, DIAPH3, ERBB2-MIR125A, ESR1, GSTM1-NFE2L2, MKI67, NDC80, RFC4, RRM2, and SCUBE2; psychomotor speed $B C L 2, C E N P A, E S R 1, M K I 67$, and PGR; verbal memory AURKA, BCL2, CCNB1, CD68, CENPA, CTSL2, DIAPH3, ESR1, ESR1-CCDC170, GSTM1, MYBL2, NDC80, ORC6, and $P G R$; visual memory - BAG1, BCL2, CCNB1, DIAPH3, ESR1, GSTM1, MYBL2, PGR, and RRM2; and visual working memory - AURKA, BAG1, BIRC5, CCNB1, CD68, DIAPH3, ESR1, GRB7, GSTM1, MELK, MYBL2, and $P G R$. All GRSs were found to be significantly $(P<0.001)$ related to the respective domain score (Table 4). Reported associations were all positive, such that as GRS increased (ie, protection), cognitive function performance score improved (Figure 1).

\section{Discussion}

\section{Individual candidate genes}

In this first study exploring relationships among polymorphisms in biologically plausible BC-related candidate genes, we report significant relationships between performance on at least one cognitive function composite domain and one or more polymorphisms of all genes evaluated, with the exception of 
Table 4 GRS and cognitive performance results

\begin{tabular}{|c|c|c|c|c|}
\hline $\begin{array}{l}\text { Cognitive } \\
\text { function } \\
\text { composite } \\
\text { domain } \\
\end{array}$ & $\begin{array}{l}\text { Gene SNP } \\
\text { used in GRS } \\
\text { calculation }\end{array}$ & $\begin{array}{l}\text { b }_{\text {GRS }} \\
P_{\text {-value }}\end{array}$ & $R^{2}$ & $\begin{array}{l}R^{2} \\
\text { change } \\
\text { for GRS }\end{array}$ \\
\hline Attention & $M I R I 25 A$ & 0.4665 & 0.2593 & 0.066 \\
\hline \multirow[t]{7}{*}{$(n=201)$} & rs 12976445 & $P<0.00 I$ & & \\
\hline & ESRI rs2347867 & & & \\
\hline & ESRI rs30203I4 & 0.48 & & \\
\hline & ESRI rs6557I7I & $P<0.00 I$ & & \\
\hline & ESRI rs985694 & & & \\
\hline & MYBL2 rs2070235 & & & \\
\hline & SCUBE2 rs6486I25 & & & \\
\hline Concentration & AURKA rs 1047972 & 0.5098 & 0.2495 & 0.189 \\
\hline \multirow[t]{20}{*}{$(n=177)$} & $B C L 2$ rs 9807663 & $P<0.00 I$ & & \\
\hline & CCNBI rs 164390 & & & \\
\hline & CCNBI rs350099 & 0.5358 & & \\
\hline & CENPA rs38065I7 & $P<0.00 I$ & & \\
\hline & DIAPH3 rs4547237 & & & \\
\hline & ESRI rs488I33 & & & \\
\hline & ESRI rs7767I43 & & & \\
\hline & ESRI rs9104I6 & & & \\
\hline & ESRI rs9397456 & & & \\
\hline & $C C D C I 70$ & & & \\
\hline & rs 12662670 & & & \\
\hline & $C C D C I 70$ & & & \\
\hline & rs3734805 & & & \\
\hline & $C C D C I 70$ & & & \\
\hline & rs37573I8 & & & \\
\hline & $C C D C I 70$ & & & \\
\hline & rs6929|37 & & & \\
\hline & GRB7 rs9910678 & & & \\
\hline & MELK rs I 0973007 & & & \\
\hline & PGR rs 10895068 & & & \\
\hline Executive & BAGI rs706118 & 0.3526 & 0.4296 & 0.204 \\
\hline function & $B C L 2$ rs I564483 & $P<0.00$ I & & \\
\hline \multirow[t]{22}{*}{$(n=137)$} & BCL2 rs4987853 & & & \\
\hline & CCNBI rs 164390 & 0.3589 & & \\
\hline & CCNBI rs350099 & $P<0.00 I$ & & \\
\hline & CCNBI rs350I04 & & & \\
\hline & CTSL2 rs436I859 & & & \\
\hline & DIAPH3 rsI337652 & & & \\
\hline & DIAPH3 rs4547237 & & & \\
\hline & $E S R I$ rs2234693 & & & \\
\hline & ESRI rs488I33 & & & \\
\hline & ESRI rs7761846 & & & \\
\hline & $E S R I$ rs82742I & & & \\
\hline & $C C D C I 70$ & & & \\
\hline & rs37573I8 & & & \\
\hline & GSTMI rs4I 2543 & & & \\
\hline & MELK rsI0973007 & & & \\
\hline & MELK rs2250340 & & & \\
\hline & MYBL2 rsII 556379 & & & \\
\hline & $P G R$ rs 1042838 & & & \\
\hline & PGR rs 474320 & & & \\
\hline & PGR rs484389 & & & \\
\hline & PGR rs608995 & & & \\
\hline & SCUBE2 rs6486I 25 & & & \\
\hline Mental & $B C L 2$ rs 1564483 & 0.504 & 0.4712 & 0.224 \\
\hline flexibility & $B C L 2$ rs4987853 & $P<0.00 I$ & & \\
\hline$(n=154)$ & DIAPH3 rsI337652 & & & \\
\hline
\end{tabular}

Table 4 (Continued)

\begin{tabular}{|c|c|c|c|c|}
\hline \multirow[t]{15}{*}{$\begin{array}{l}\text { Cognitive } \\
\text { function } \\
\text { composite } \\
\text { domain } \\
\end{array}$} & $\begin{array}{l}\text { Gene SNP } \\
\text { used in GRS } \\
\text { calculation }\end{array}$ & $\begin{array}{l}\mathbf{b}_{\mathrm{GRS}} \\
P_{\text {-value }}\end{array}$ & $R^{2}$ & $\begin{array}{l}R^{2} \\
\text { change } \\
\text { for GRS }\end{array}$ \\
\hline & $M I R I 25 A$ & 0.5383 & & \\
\hline & rs 12976445 & $P<0.001$ & & \\
\hline & ESRI rs2347867 & & & \\
\hline & ESRI rs6557I7I & & & \\
\hline & ESRI rs985694 & & & \\
\hline & NFE2L2 & & & \\
\hline & rs35652I24 & & & \\
\hline & MKI67 rsI0732438 & & & \\
\hline & MYBL2 rsII 556379 & & & \\
\hline & NDC80 rs 12408485 & & & \\
\hline & NDC80 rs2292274 & & & \\
\hline & RFC4 rs 1354091 & & & \\
\hline & RRM2 rs I I 38729 & & & \\
\hline & SCUBE2 rs6486I 25 & & & \\
\hline \multirow{9}{*}{$\begin{array}{l}\text { Psychomotor } \\
\text { speed }(n=\mid 81)\end{array}$} & $B C L 2$ rs494II95 & 0.7265 & 0.2527 & 0.093 \\
\hline & BCL2 rs 956572 & $P<0.00 \mathrm{I}$ & & \\
\hline & CENPA rs3806518 & & & \\
\hline & $E S R I$ rs 2347867 & 0.6674 & & \\
\hline & ESRI rs488I33 & $P<0.001$ & & \\
\hline & ESRI rs9322331 & & & \\
\hline & ESRI rs9340799 & & & \\
\hline & MKI67 rsI0732438 & & & \\
\hline & PGR rs568I57 & & & \\
\hline \multirow{30}{*}{$\begin{array}{l}\text { Verbal } \\
\text { memory } \\
(n=146)\end{array}$} & AURKA rsI6979877 & 0.3406 & 0.5048 & 0.209 \\
\hline & $B C L 2$ rs2279II5 & $P<0.001$ & & \\
\hline & $B C L 2$ rs4987852 & & & \\
\hline & BIRC5 rs3764383 & 0.3401 & & \\
\hline & CCNBI rsI64390 & $P<0.001$ & & \\
\hline & CCNBI rs350099 & & & \\
\hline & CCNBI rs350I04 & & & \\
\hline & CD68 rs9901673 & & & \\
\hline & CENPA rs38065I 8 & & & \\
\hline & CTSL2 rsI6919034 & & & \\
\hline & DIAPH3 rs4547237 & & & \\
\hline & ESRI rsI04849I9 & & & \\
\hline & ESRI rsI2665044 & & & \\
\hline & $E S R I$ rs294I740 & & & \\
\hline & ESRI rs $488 \mid 33$ & & & \\
\hline & ESRI rs77275268 & & & \\
\hline & ESRI rs7767|43 & & & \\
\hline & ESRI rs9383938 & & & \\
\hline & ESRI rs93974355 & & & \\
\hline & $C C D C I 70$ & & & \\
\hline & rs3734805 & & & \\
\hline & $C C D C I 70$ & & & \\
\hline & rs3757318 & & & \\
\hline & GSTMI rs4I 2543 & & & \\
\hline & MYBL2 rs2070235 & & & \\
\hline & MYBL2 rs619289 & & & \\
\hline & NDC80 rs2292274 & & & \\
\hline & ORC6 rs33994299 & & & \\
\hline & PGR rs484389 & & & \\
\hline & PGR rs568I57 & & & \\
\hline \multirow{3}{*}{$\begin{array}{l}\text { Visual memory } \\
(n=165)\end{array}$} & BAGI rs706II8 & 0.7477 & 0.3167 & 0.148 \\
\hline & $B C L 2$ rs 1564483 & $P<0.001$ & & \\
\hline & CCNBI rs350104 & & & \\
\hline
\end{tabular}


Table 4 (Continued)

\begin{tabular}{|c|c|c|c|c|}
\hline $\begin{array}{l}\text { Cognitive } \\
\text { function } \\
\text { composite } \\
\text { domain }\end{array}$ & $\begin{array}{l}\text { Gene SNP } \\
\text { used in GRS } \\
\text { calculation }\end{array}$ & $\begin{array}{l}\mathbf{b}_{\mathrm{GRS}} \\
\boldsymbol{P} \text {-value }^{\mathrm{a}}\end{array}$ & $R^{2}$ & $\begin{array}{l}R^{2} \\
\text { change } \\
\text { for GRS }\end{array}$ \\
\hline & 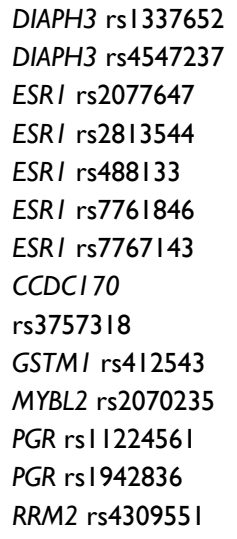 & $\begin{array}{l}0.6078 \\
P<0.001\end{array}$ & & \\
\hline $\begin{array}{l}\text { Visual working } \\
\text { memory } \\
(n=154)\end{array}$ & 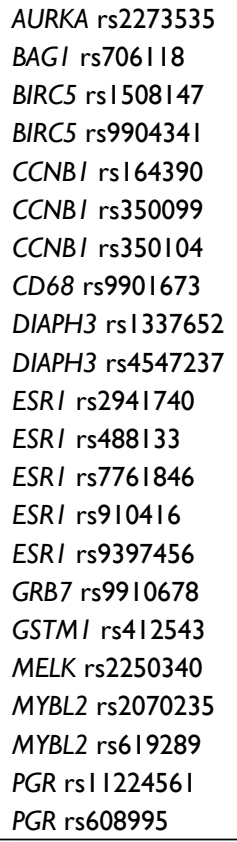 & $\begin{array}{l}0.4198 \\
P<0.001 \\
0.4131 \\
P<0.001\end{array}$ & 0.47 & $0.24 I$ \\
\hline
\end{tabular}

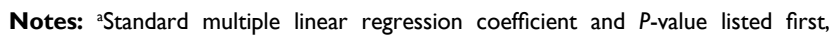
robust (generated using Huber weighting and biweighting iterations) multiple linear regression coefficient and $P$-value listed subsequently. Model $R^{2}$ and $R^{2}$ change reported from standard multiple linear regression models. Participants missing genetic data necessary for completion of a GRS calculation were not included in the GRS analysis. All regression models adjusted for age, estimated verbal intelligence, levels of depressive symptoms, anxiety, fatigue, pain, and prescribed treatment group. Abbreviations: GRS, genetic risk/protection score; SNP, single-nucleotide polymorphism.

CMC2, MMP11, and RACGAP1. Significant findings related to the candidate genes found most broadly to impact cognitive function performance across multiple domains, specifically ESR1, CCDC170, PGR, CCNB1, MYBL2, BCL2, GSTM1, and $D I A P H 3$, are discussed in detail in the following sections.

\section{$E S R I$ and $C C D C I 70$}

The ESR 1 gene encodes an estrogen receptor. Polymorphisms in ESR1 have been previously associated with cognitive outcomes, including functioning, impairment, and Alzheimer's disease. ${ }^{43}$ We found that performance on every cognitive domain was related to ESR 1 polymorphisms through either main effects and/or interaction effects. The most global associations with a single $E S R 1$ polymorphism occurred with an intronic upstream variant - rs488133. The effects of this polymorphism on cognitive function performance were different by domain and study cohort: rs488133-CT+TT contributed positively to executive function and psychomotor speed performance in all study participants. rs488133-CT+TT negatively impacted concentration performance in HCs, but positively impacted concentration performance in women with $\mathrm{BC}$ prescribed AO. In contrast, rs488133-CT+TT positively impacted memory performance in $\mathrm{HCs}$, but negatively impacted memory performance in women with $\mathrm{BC}$ prescribed AO. In addition, while reported in other investigations of middle-aged and older women, we did not observe global cognitive impairment trends or memory deficits related to two well-studied polymorphisms in exon 1 of ESR1 named for the respective restriction enzyme-recognition sites: PvuII (rs2234693) and Xbal (rs9340799). ${ }^{44-47}$

Polymorphisms in $C C D C 170$, the upstream neighbor of ESR1, were included in this study to represent more fully variability in ESR1. Associations between CCDC170 polymorphisms and $\mathrm{BC}$ susceptibility, progression, and survival have been reported..$^{25,26,48-50}$ In addition, ESR I-CCDC170 chromosomal rearrangements have been associated with more aggressive estrogen receptor-positive $\mathrm{BCs} .{ }^{51}$ While the function of $C C D C 170$ is unknown, and no studies to date have investigated associations between $C C D C 170$ polymorphisms and cognitive phenotypes, results from this analysis, in which possession of one or more CCDC170 MAs in four (rs12662670, rs3734805, rs3757318, and rs6929137) of the five SNPs evaluated was related to poorer concentration performance in all study participants, suggest that variation in $C C D C 170$ plays an important role in concentration.

\section{PGR}

Progesterone receptors, encoded by $P G R$, are expressed throughout the brain in every neural cell type.${ }^{52}$ Henderson et al found that progesterone concentrations were significantly and positively related to global cognition and verbal memory performance in healthy women less than 6 years since menopause. ${ }^{53}$ Moreover, Voytko et al found that estrogen plus progesterone improved executive function and attention performance in surgically menopausal monkeys. ${ }^{54}$ For executive function performance, we observed significant interactions between multiple $P G R$ polymorphisms and study cohorts. In all instances, possession of $P G R$ rs 1042838-GT+TT, 

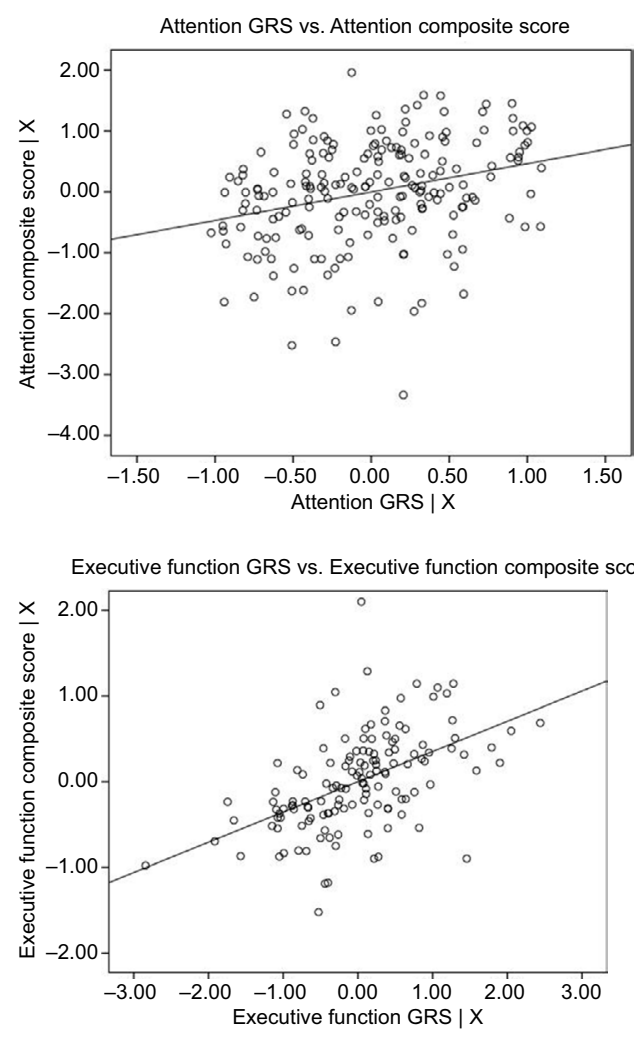

Psychomotor speed GRS vs. Psychomotor speed composite score
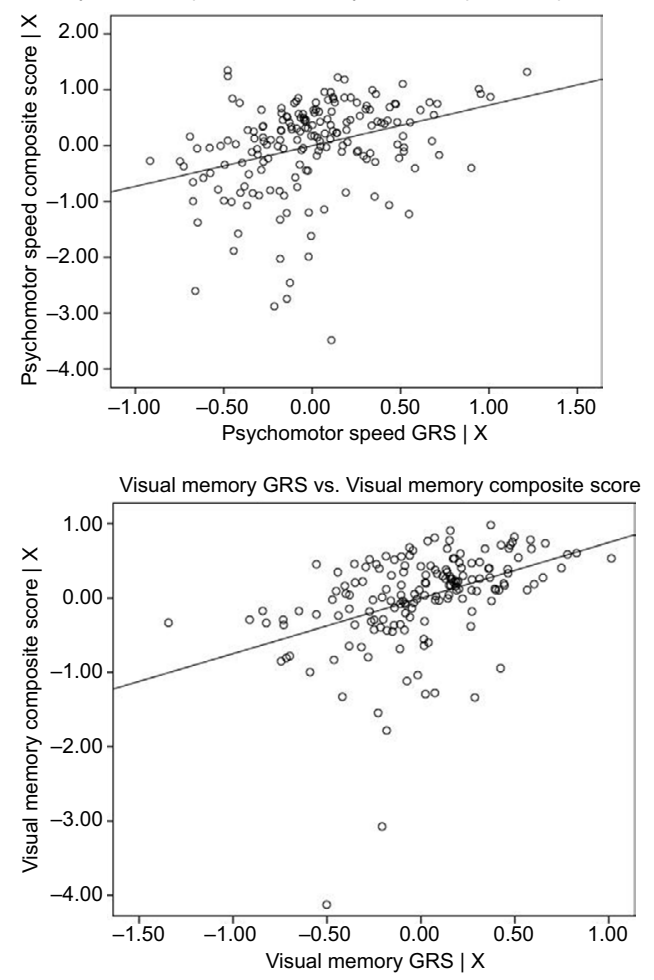

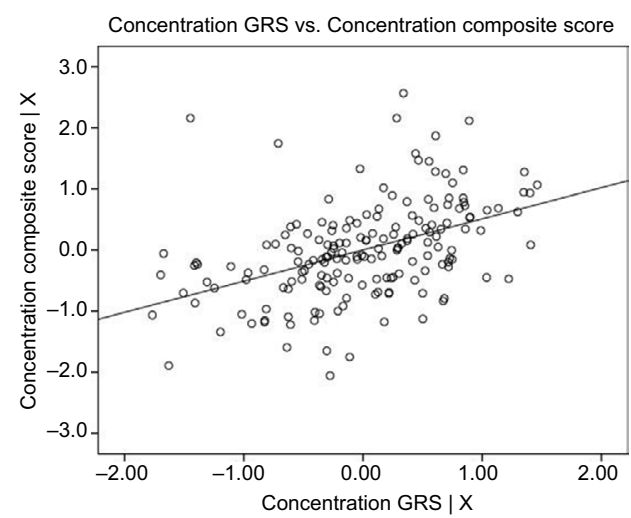

Mental flexibility GRS vs. Mental flexibility composite score
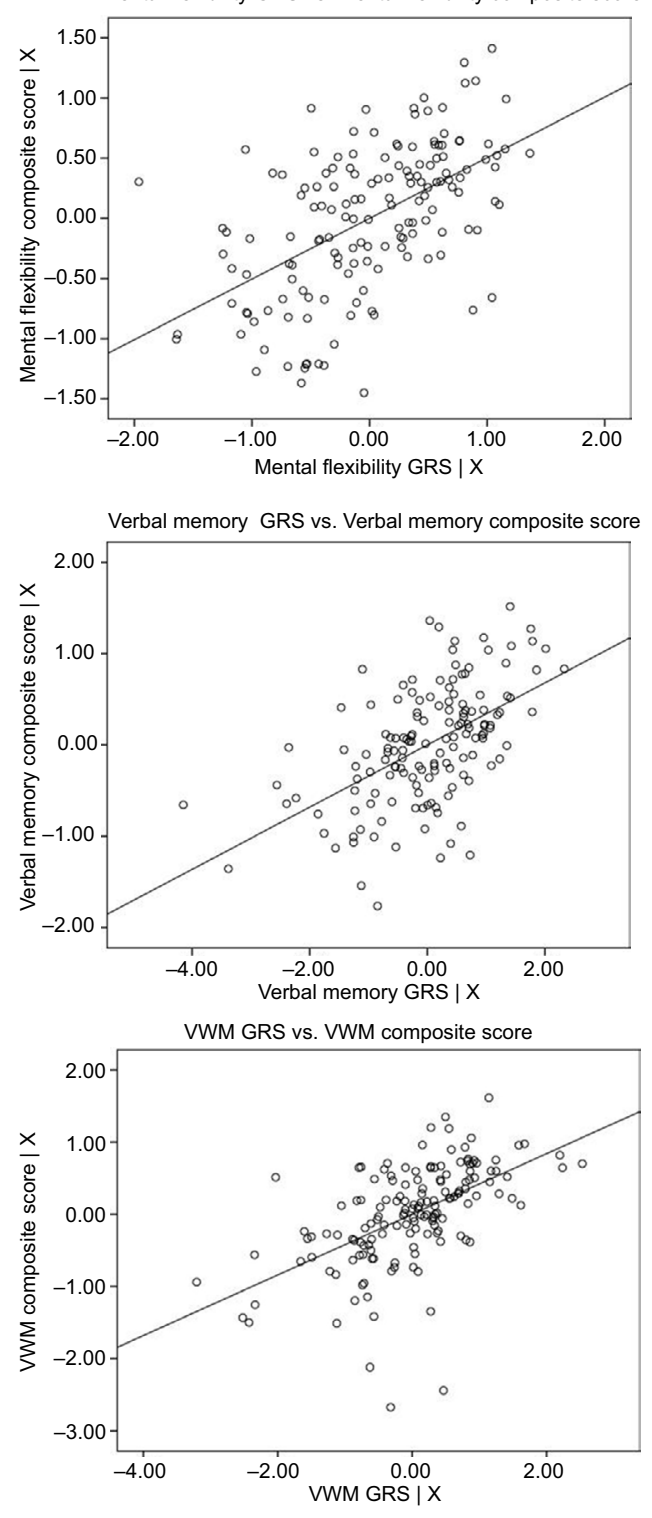

Figure I GRS by cognitive function composite score-partial regression plots.

Notes: $\mathrm{X}=$ age, estimated verbal intelligence, levels of depressive symptoms, anxiety, fatigue, pain, and prescribed treatment. Figure generated using SPSS version 24 (IBM Corporation, Armonk, NY, USA).

Abbreviations: GRS, genetic risk/protection score; VWM, Visual Working Memory. 
$P G R$ rs474320-TA+AA, $P G R$ rs484389-TC+CC, or $P G R$ rs608995-AT+TT genotypes contributed positively to executive function-performance scores in HCs. When we looked at the interaction of these MAs within the context of BC, we saw the opposite effect: the combination of possession of one or more MAs and membership in a BC cohort was found to impact scores negatively, offsetting the positive SNP main effects and contributing an overall negative input to executive function performance in multiple instances.

The first SNP, rs1042838 (Val660Leu, G>T), is a missense polymorphism in exon 4 that is in linkage disequilibrium with rs1042839 (His770His, C>T), a silent polymorphism in exon 5, and a 320 bp Alu-element insertion at intron G; collectively, these polymorphisms form a variant haplotype called PROGINS. While the functional consequences remain unclear, the PROGINS allele has been associated with increased breast and ovarian cancer risk. ${ }^{55-59}$ Also evaluated in this study was rs474320, an intronic variant reported to be in tight linkage with PROGINS, ${ }^{60}$ and rs 1042839, which is tightly linked to rs1042838. Both SNPs were found to be significant and as expected: rs1042839 generated very similar results to rs1042838; discrepancies in call rate may account for the differences in significance. The remaining significant SNPs, rs484389 and rs608995, are located in the UTR3' of $P G R$. Taken together, these findings indicate that variation in regulation of progesterone receptors may be associated with executive function performance, and furthermore that the polymorphic impact on performance may vary in the systemic environment of a healthy individual compared to that of an individual diagnosed with BC.

\section{CCNBI}

CCNB1 encodes a cell-cycle regulatory protein important in mitosis. ${ }^{61}$ Because expression levels from this gene are used in three of five of the prognostic multigene-expression profiles for $\mathrm{BC}$ from which candidate genes were identified, CCNB1 was one of our top candidates for investigation of study hypotheses. ${ }^{5}$ Significant interactions were reported with study cohorts for three functional polymorphisms - rs 164390 $(102 \mathrm{G}>\mathrm{T})$, rs350099 (-957C>T), and rs350104 (-457C>T) located in the promotor region of $C C N B 1$ and memory and executive function performance. In general, we found that possession of rs164390GT+TT or rs350099CT+CC genotypes contributed positively to performance scores in $\mathrm{HCs}$ but close to zero or negatively in women with BC. The opposite contribution was observed for rs350104CT+CC genotypes. The genotypes associated with poorer cognitive performance in the cohorts of women with BC, rs164390-GT+TT,
rs350099-CT+CC, and rs350104-TT, are all hypothesized to lead to lower levels of $C C N B 1$ expression via reduced recruitment of transcription factors to the promotor region of the gene. ${ }^{62}$ This result is contradictory to anticipated findings, as higher cyclin B levels in breast tissue are associated with more severe cancer phenotypes. ${ }^{63,64}$ In addition, cyclin B levels were reported to be upregulated in autopsy hippocampal tissue in individuals with neuropathological Alzheimer's disease and clinical dementia compared to individuals with normal aging. ${ }^{65}$ Nevertheless, the consistency of findings across three variants all theorized to impact expression in the same direction lends support to these associations. We would like to point out that one or more polymorphisms in the four other genes represented in three prognostic multigeneexpression profiles for $\mathrm{BC}-C E N P A, M E L K, M Y B L 2$, and ORC6 - were associated with performance on at least one domain.

\section{MYBL2 and BCL2}

MYBL2 encodes a nuclear protein, B-Myb, involved in cellcycle progression and promotion of cell survival through activation of antiapoptotic genes. ${ }^{61,66}$ However, overexpression of B-Myb in certain settings induces apoptosis, and has been reported to contribute to neuronal cell death. ${ }^{66-69}$ We found significant relationships with two missense polymorphisms in MYBL2: rs11556379 (Ile624Met, C>G) and rs2070235 (Ser427Gly, A>G). The MAs of these polymorphisms have been reported to alter protein conformation, impair regulation of downstream targets, decrease antiapoptotic activity, and reduce cancer risk. ${ }^{70}$ Interestingly, for all study participants, rs2070235-AG+GG genotypes contributed positively to attention and negatively to memory-performance scores, while rs 1 1556379-CG+GG genotypes contributed positively to mental flexibility-performance scores. We also reported a significant interaction related to executive function, where rs11556379-CG+GG genotypes had the opposite impact on performance in $\mathrm{HCs}$ (positive contribution to scores) and women with BC (negative contribution to scores).

Additionally, we report associations between polymorphisms in a gene regulated by $M Y B L 2$ that is also involved in apoptosis, BCL2, and concentration, executive function, mental flexibility, psychomotor speed, verbal memory, and visual memory performance. BCL2 expression has been associated with prognostication of disease-free survival, overall survival, and recurrence in BC. ${ }^{71-78}$ Moreover, normal breast tissue from women with $\mathrm{BC}$ was reported to display higher levels of $B C L 2$ expression than breast tissue from women with no evidence of cancer. ${ }^{79}$ In relation to neurologic 
phenotypes, polymorphisms in BCL2 have been found to impact outcomes after traumatic brain injury and have been associated with hippocampal volume. ${ }^{80,81}$

\section{GSTM I}

One of the functional polymorphisms located in the promoter region of GSTM1, rs412543(-498C $>\mathrm{G})$, was found to be important for memory and executive function performance. GSTM1 encodes an enzyme with antioxidant properties that detoxifies electrophilic compounds, including carcinogens, drugs, and environmental toxins, throughout the body. ${ }^{61} \mathrm{By}$ decreasing the binding capability of the transcription factor AP2 to the GSTM1-promoter region, the $\mathrm{G}$ allele has been reported to decrease GSTM1 transcription by $30 \%-40 \%$ compared to the $\mathrm{C}$ allele. ${ }^{82}$ Both decreased and enhanced (attributed to counterproductive depletion of glutathione) GSTM1 expression has been associated with increased BC risk. ${ }^{82-84}$ We found that rs412543-GG+CG and hypothesized decreased GSTM1 expression contributed negatively to executive function and memory performance in all study participants. However, we also found positive interaction effects between rs412543-GG+CG and BC cohort related to verbal and visual working memory. While the mechanism is unclear, the paradoxical quality of GSTM1 under- and overexpression combined with study results suggests that decreased or moderate GSTM1 expression may be beneficial to certain aspects of cognitive function in women with $\mathrm{BC}$. Considering the detoxification properties of GSTM1, further evaluation of cognitive decline over time in women with BC receiving adjuvant chemotherapy and/or antiestrogen therapy is recommended.

\section{DIAPH3}

Variation in the two upstream intronic polymorphisms selected to represent DIAPH3, rs 1337652 and rs4547237, were associated with performance for multiple domains as well. DIAPH3 is involved in actin remodeling and regulation of cell movement and adhesion. ${ }^{61}$ DIAPH3 downregulation and silencing has been associated with metastatic disease due to loss of normal gene function and acquisition of an amoeboid cancer-cell phenotype. ${ }^{85}$ Evidence also suggests that DIAPH3 is critical to brain development and is involved in cell migration, the formation of dendrites and axons, axon guidance, and synaptic activity ${ }^{86}$

\section{CMC2, MMPII, and RACGAPI}

Three candidate genes were not significantly associated with pretreatment cognitive performance in this study. While genes with significant findings from our analysis are represented by multiple functional and/or tagging SNPs and are well described in the literature, it is notable that the three genes not found to be significant are less well represented in the literature and the HapMap database. Single SNPs, rs 131451 and rs7303531, were included in the analysis for MMP11 and $R A C G A P 1$, respectively. Both SNPs are upstream variants. No associations have been reported between MMP11 or $R A C G A P 1$ and cognitive phenotypes in the literature. $C M C 2$ is an even more poorly described and studied gene, with reported involvement in cytochrome $\mathrm{C}$ oxidase activity. ${ }^{87}$ Two upstream (rs1025065 and rs1981867) polymorphisms and one downstream (rs9936489) polymorphism were identified using the Phase III HapMap database based on National Center for Biotechnology Information gene location (Chr16: $80975802 . .81006897)$, as $C M C 2$ is not a displayed gene in HapMap. We must be mindful that our analysis is limited to current information known about these genes and polymorphisms, and thus these genes cannot be ruled out as important to understanding cognitive function within the context of BC.

\section{Genetic risk/protection scores}

Because of the complexity of $\mathrm{BC}$ as a disease and cognitive function as a phenotype, we calculated weighted GRSs for each domain to evaluate the collective effect of possession of multiple risk or protective MAs of genes used to clinically evaluate the biology of BC. Every GRS was significantly $(P<0.001)$ and positively associated with its respective domain. When the GRSs were added as predictors to regression models, including age, estimated verbal intelligence, levels of depressive symptoms, anxiety, fatigue, pain, and prescribed treatment group, the explained variance $\left(R^{2}\right)$ increased by 0.066 to 0.244 for each domain. This substantial increase in $R^{2}$ speaks to both the importance of host variation in genes used to evaluate clinically the biology of $\mathrm{BC}$ to pretreatment cognitive performance and the use of multiple common variants, plus personal and environmental factors, to model a complex phenotype.

\section{Limitations and future directions}

Small sample sizes limited our ability to conduct genetic analyses by genotype, rather than by the presence or absence of one or more MAs; therefore, we were unable to evaluate gene-dosage effects. In addition, the sample was comprised of postmenopausal women with hormone receptor-positive, early stage BC who were primarily Caucasian; therefore, the generalizability of study findings to premenopausal women, hormone-negative, different-stage $\mathrm{BCs}$, or more diverse 
patient populations is unknown. The number of statistical tests completed as part of this exploratory study and possible inflation of type I error should also be acknowledged; all reported results will need to be confirmed in future independent studies. Limitations related to the prioritization and inclusion of select candidate genes has been discussed previously. ${ }^{5}$

While biomarkers of host DNA and cognitive performance are advantageous for a number of reasons, including the stability and tissue nonspecificity of DNA polymorphisms, associations with gene-expression and protein levels should also be conducted, as some of the most prominent findings from this study were related to polymorphisms with known functional consequences or located in regulatory regions. We postulate that cognitive performance variability in women with BC may be at least partially driven by tumor-gene expression and corresponding protein levels. Longitudinal studies that include cognitive assessment prior to primary surgery would be ideal for evaluation of the effect of tumor-gene expression, as well as changes in gene expression due to tumor removal and treatment of primary and secondary cancer sites, on variability in cognitive performance. Significant relationships from tumor gene-expression studies are advantageous for different reasons; namely, they could directly expand the clinical utility of currently marketed prognostic multigene-expression profiles for BC. Future analyses should also investigate the effect of polymorphisms in genes used to clinically evaluate the biology of $\mathrm{BC}$ and tumor-expression levels on cognitive function throughout and following adjuvant chemotherapy and/or antiestrogentherapy regimens.

\section{Conclusion}

In summary, the objective of this study was to explore the hypothesis that host variation in candidate genes involved in $\mathrm{BC}$ development and prognosis is associated with variability in the presence and/or severity of alterations in pretreatment cognitive performance among postmenopausal women diagnosed with early stage BC. Significant associations between host polymorphisms representing 25 candidate genes used to clinically evaluate the biology of BC and computed GRSs and variability in pretreatment cognitive function performance support this hypothesis and merit independent replication and further investigation into the identification of clinically relevant biomarkers for BC-related cognitive dysfunction.

\section{Acknowledgments}

This study was funded by the National Institute of Nursing Research Cognitive Function and Breast Cancer: Genomics and Disease Characteristics (F31NR014590) and Targeted Research and Academic Training of Nurses in Genomics (T32NR009759), National Cancer Institute Long Term Trajectory of Cognitive Function Related to Anastrozole Use in Women (R01CA107408), American Cancer Society Doctoral Degree Scholarship in Cancer Nursing (DSCN14-076-01-SCN), Oncology Nursing Society Foundation, a Sigma Theta Tau International Eta Chapter Research Award, a Nightingale Awards of Pennsylvania PhD degree scholarship, and a University of Pittsburgh School of Nursing Ruth and Bill Finke PhD Student research award.

\section{Disclosure}

The authors report no conflicts of interest in this work.

\section{References}

1. Runowicz CD, Leach CR, Henry NL, et al. American Cancer Society/ American Society of Clinical Oncology breast cancer survivorship care guideline. J Clin Oncol. 2016;34(6):611-635.

2. Wefel JS, Kesler SR, Noll KR, Schagen SB. Clinical characteristics, pathophysiology, and management of noncentral nervous system cancer-related cognitive impairment in adults. CA Cancer J Clin. 2015;65(2):123-138.

3. Ahles TA, Root JC, Ryan EL. Cancer- and cancer treatment-associated cognitive change: an update on the state of the science. J Clin Oncol. 2012;30(30):3675-3686.

4. Ahles TA, Saykin AJ. Candidate mechanisms for chemotherapy-induced cognitive changes. Nat Rev Cancer. 2007;7(3):192-201.

5. Koleck TA, Conley YP. Identification and prioritization of candidate genes for symptom variability in breast cancer survivors based on disease characteristics at the cellular level. Breast Cancer (Dove Med Press). 2016;8:29-37.

6. Koleck TA, Bender CM, Sereika SM, et al. Apolipoprotein E genotype and cognitive function in postmenopausal women with early-stage breast cancer. Oncol Nurs Forum. 2014;41(6):E313-E325.

7. Ahles TA, Saykin AJ, Noll WW, et al. The relationship of APOE genotype to neuropsychological performance in long-term cancer survivors treated with standard dose chemotherapy. Psychooncology. 2003;12(6):612-619.

8. Ahles TA, Li Y, McDonald BC, et al. Longitudinal assessment of cognitive changes associated with adjuvant treatment for breast cancer: the impact of APOE and smoking. Psychooncology. 2014; 23(12):1382-1390.

9. Lengacher CA, Reich RR, Kip KE, et al. Moderating effects of genetic polymorphisms on improvements in cognitive impairment in breast cancer survivors participating in a 6-week mindfulness-based stress reduction program. Biol Res Nurs. 2015;17(4):393-404.

10. Ng T, Teo SM, Yeo HL, et al. Brain-derived neurotrophic factor genetic polymorphism (rs6265) is protective against chemotherapy-associated cognitive impairment in patients with early-stage breast cancer. Neuro Oncol. 2016;18(2):244-251.

11. Small BJ, Rawson KS, Walsh E, et al. Catechol-O-methyltransferase genotype modulates cancer treatment-related cognitive deficits in breast cancer survivors. Cancer. 2011;117(7):1369-1376.

12. Koleck TA, Bender CM, Sereika SM, et al. Polymorphisms in DNA repair and oxidative stress genes associated with pre-treatment cognitive function in breast cancer survivors: an exploratory study. Springerplus. 2016;5:422.

13. Bender CM, Merriman JD, Gentry AL, et al. Patterns of change in cognitive function with anastrozole therapy. Cancer. 2015;121(15):2627-2636.

14. Jerevall PL, Ma XJ, Li H, et al. Prognostic utility of HOXB13:IL17BR and molecular grade index in early-stage breast cancer patients from the Stockholm trial. Br J Cancer. 2011;104(11):1762-1769. 
15. Tutt A, Wang A, Rowland C, et al. Risk estimation of distant metastasis in node-negative, estrogen receptor-positive breast cancer patients using an RT-PCR based prognostic expression signature. BMC Cancer. 2008;8:339.

16. Paik S, Shak S, Tang G, et al. A multigene assay to predict recurrence of tamoxifen-treated, node-negative breast cancer. $N \mathrm{Engl} \mathrm{J} \mathrm{Med}$. 2004;351(27):2817-2826.

17. Paik S, Tang G, Shak S, et al. Gene expression and benefit of chemotherapy in women with node-negative, estrogen receptor-positive breast cancer. J Clin Oncol. 2006;24(23):3726-3734.

18. Parker JS, Mullins M, Cheang MC, et al. Supervised risk predictor of breast cancer based on intrinsic subtypes. J Clin Oncol. 2009;27(8): 1160-1167.

19. van't Veer LJ, Dai H, van de Vijver MJ, et al. Gene expression profiling predicts clinical outcome of breast cancer. Nature. 2002;415(6871):530-536

20. Buyse M, Loi S, van't Veer L, et al. Validation and clinical utility of a 70-gene prognostic signature for women with node-negative breast cancer. J Natl Cancer Inst. 2006;98(17):1183-1192.

21. Lehmann TP, Korski K, Ibbs M, Zawierucha P, Grodecka-Gazdecka S, Jagodziński PP. rs12976445 Variant in the pri-miR-125a correlates with a lower level of hsa-miR-125a and ERBB2 overexpression in breast cancer patients. Oncol Lett. 2013;5(2):569-573.

22. Hu Y, Liu CM, Qi L, et al. Two common SNPs in pri-miR-125a alter the mature miRNA expression and associate with recurrent pregnancy loss in a Han-Chinese population. RNA Biol. 2011;8(5):861-872.

23. Barzan D, Veldwijk MR, Herskind C, et al. Comparison of genetic variation of breast cancer susceptibility genes in Chinese and German populations. Eur J Hum Genet. 2013;21(11):1286-1292.

24. Han J, Jiang T, Bai H, et al. Genetic variants of $6 \mathrm{q} 25$ and breast cancer susceptibility: a two-stage fine mapping study in a Chinese population. Breast Cancer Res Treat. 2011;129(3):901-907.

25. Hein R, Maranian M, Hopper JL, et al. Comparison of 6q25 breast cancer hits from Asian and European genome wide association studies in the Breast Cancer Association Consortium (BCAC). PLoS One 2012;7(8):e42380.

26. Stevens KN, Vachon CM, Lee AM, et al. Common breast cancer susceptibility loci are associated with triple-negative breast cancer. Cancer Res. 2011;71(19):6240-6249.

27. Fletcher O, Johnson N, Orr N, et al. Novel breast cancer susceptibility locus at 9q31.2: results of a genome-wide association study. J Natl Cancer Inst. 2011;103(5):425-435.

28. Yu B, Lin H, Yang L, et al. Genetic variation in the Nrf2 promoter associates with defective spermatogenesis in humans. J Mol Med. 2012;90(11):1333-1342.

29. Miller SA, Dykes DD, Polesky HF. A simple salting out procedure for extracting DNA from human nucleated cells. Nucleic Acids Res. 1988;16(3):1215.

30. DNA Genotek. Laboratory protocol for manual purification of DNA from whole sample. 2015. Available from: http://www.dnagenotek.com/ US/pdf/PD-PR-015.pdf. Accessed December 14, 2016.

31. Robbins T, James M, Owen A, Sahakian B, McInnes L, Rabbitt P. Cambridge Neuropsychological Test Automated Battery (CANTAB): a factor analytic study of a large sample of normal elderly volunteers. Dementia. 1994;5(5):266-281.

32. Lafayette Clinical Instruments Company. Lafayette Clinical Repeatable Neuropsychological Battery. Sagamore (IN): Lafayette Clinical Instruments Company; 1989.

33. Delis DC, Kaplan E, Kramer JH. Delis-Kaplan (D-KEFS) Executive Function System, Examiners Manual. San Antonio: Psychological Corporation; 2001.

34. Klove H. Clinical neuropsychology. Med Clin North Am. 1963;47: 1647-1658.

35. Wechsler D. The Wechsler Memory Scale: Revised Manual. San Antonio: Psychological Corporation; 1998.

36. Rey A. L'examen psychologique dans les cas d'encephalopathie traumatique (les problems) [The psychological examination in cases of traumatic encephalopathy: problems]. Arch Psychol. 1941;28:215-285. French.
37. Cockburn J, Smith PT. Correlates of everyday memory among residents of Part III homes. Br J Clin Psychol. 1993;32(Pt 1):75-77.

38. Osterrieth PA. Le test de copie d'une figure complexe: contribution a l'etude de la perception et de la memoire [Test of copying a complex figure: contribution to the study of perception and memory]. Arch Psychol. 1944;30:206-356. French.

39. Nelson H. The National Adult Reading Test (NART): Test Manual. Windsor, UK: NFER-Nelson; 1982.

40. Beck AT, Steer RA, Brown GK. Beck Depression Inventory-II. San Antonio: Psychological Corporation; 1996.

41. McNair D, Lorr M, Droppleman LF. EdITS Manual for the Profile of Mood States (POMS). San Diego: Educational and Industrial Testing Service; 1992.

42. Cleeland CS. Measurement of pain by subjective report. In: Chapman CR, Loeser JD, editors. Advances in Pain Research and Therapy. Vol 12. New York: Raven Press; 1989:391-403.

43. Sundermann EE, Maki PM, Bishop JR. A review of estrogen receptor alpha gene (ESR1) polymorphisms, mood, and cognition. Menopause. 2010;17(4):874-886.

44. Yaffe K, Lindquist K, Sen S, et al. Estrogen receptor genotype and risk of cognitive impairment in elders: findings from the Health $\mathrm{ABC}$ study. Neurobiol Aging. 2009;30(4):607-614.

45. Bousman CA, Szoeke C, Chen K, Dennerstein L, Henderson VW, Everall IP. Oestrogen alpha-receptor variant and two-year memory decline in midlife Australian women. Neuropsychobiology. 2012;66(4): 259-265.

46. Kravitz HM, Meyer PM, Seeman TE, Greendale GA, Sowers MR. Cognitive functioning and sex steroid hormone gene polymorphisms in women at midlife. Am J Med. 2006;119(9 Suppl 1):S94-S102.

47. Yaffe K, Lui LY, Grady D, Stone K, Morin P. Estrogen receptor 1 polymorphisms and risk of cognitive impairment in older women. Biol Psychiatry. 2002;51(8):677-682.

48. Yamamoto-Ibusuki M, Yamamoto Y, Fujiwara S, et al. C6ORF97-ESR1 breast cancer susceptibility locus: influence on progression and survival in breast cancer patients. Eur J Hum Genet. 2015;23(7):949-956.

49. Hong Y, Chen XQ, Li JY, et al. Current evidence on the association between rs 3757318 of C6orf97 and breast cancer risk: a meta-analysis. Asian Pac J Cancer Prev. 2014;15(19):8051-8055.

50. Fletcher O, Johnson N, Orr N, et al. Novel breast cancer susceptibility locus at 9q31.2: results of a genome-wide association study. $J$ Natl Cancer Inst. 2011;103(5):425-435.

51. Veeraraghavan J, Tan Y, Cao XX, et al. Recurrent ESR1-CCDC170 rearrangements in an aggressive subset of estrogen-receptor positive breast cancers. Nat Commun. 2015;5:4577.

52. Brinton RD, Thompson RF, Foy MR, et al. Progesterone receptors: form and function in brain. Front Neuroendocrinol. 2008;29(2):313-339.

53. Henderson VW, St John JA, Hodis HN, et al. Cognition, mood, and physiological concentrations of sex hormones in the early and late postmenopause. Proc Natl Acad Sci U S A. 2013;110(50): 20290-20295.

54. Voytko ML, Murray R, Higgs CJ. Executive function and attention are preserved in older surgically menopausal monkeys receiving estrogen or estrogen plus progesterone. J Neurosci. 2009;29(33):10362-10370.

55. Stenzig J, Schweikert A, Piasecki A, Höppner G, Eschenhagen T, Rau T. Progesterone receptor variants associated with the PROGINS haplotype exhibit functional properties similar to those of wild-type progesterone receptor. Pharmacogenet Genomics. 2012;22(8):629-641.

56. Agoulnik IU, Tong XW, Fischer DC, et al. A germline variation in the progesterone receptor gene increases transcriptional activity and may modify ovarian cancer risk. $J$ Clin Endocrinol Metab. 2004;89(12):6340-6347.

57. Rockwell LC, Rowe EJ, Arnson K, et al. Worldwide distribution of allelic variation at the progesterone receptor locus and the incidence of female reproductive cancers. Am J Hum Biol. 2012;24(1): $42-51$.

58. Liu T, Chen L, Sun X, et al. Progesterone receptor PROGINS and $+331 \mathrm{G} /$ A polymorphisms confer susceptibility to ovarian cancer: a meta-analysis based on 17 studies. Tumor Biol. 2014;35(3):2427-2436. 
59. Romano A, Delvoux B, Fischer DC, Groothuis P. The PROGINS polymorphism of the human progesterone receptor diminishes the response to progesterone. J Mol Endocrinol. 2007;38(1-2):331-350.

60. Lee E, Hsu C, Haiman CA, et al. Genetic variation in the progesterone receptor gene and risk of endometrial cancer: a haplotype-based approach. Carcinogenesis. 2010;31(8):1392-1399.

61. NCBI Resource Coordinators. Database resources of the National Center for Biotechnology Information. Nucleic Acids Res. Epub 2014 Nov 14.

62. Silvestre-Roig C, Fernández P, Mansego ML, et al. Genetic variants in CCNB1 associated with differential gene transcription and risk of coronary in-stent restenosis. Circ Cardiovasc Genet. 2014;7(1):59-70.

63. Winters ZE, Hunt NC, Bradburn MJ, et al. Subcellular localisation of cyclin B, Cdc2 and p21(WAF1/CIP1) in breast cancer: association with prognosis. Eur J Cancer. 2001;37:2405-2412.

64. Kawamoto H, Koizumi H, Uchikoshi T. Expression of the G2-M checkpoint regulators cyclin $\mathrm{B} 1$ and $\mathrm{Cdc} 2$ in nonmalignant and malignant human breast lesions: immunocytochemical and quantitative image analyses. Am J Pathol. 1997;150(1):15-23.

65. Silva AR, Santos AC, Farfel JM, et al. Repair of oxidative DNA damage, cell-cycle regulation and neuronal death may influence the clinical manifestation of Alzheimer's disease. PLoS One. 2014;9(6):e99897.

66. Sala A. B-MYB, a transcription factor implicated in regulating cell cycle, apoptosis and cancer. Eur J Cancer. 2005;41(16):2479-2484.

67. Lui DX, Nath N, Chellappan SP, Greene LA. Regulation of neuron survival and death by $\mathrm{p} 130$ and associated chromatin modifiers. Genes Dev. 2005;19(6):719-732.

68. Liu DX, Biswas SC, Greene LA. B-Myb and C-Myb play required roles in neuronal apoptosis evoked by nerve growth factor deprivation and DNA damage. J Neurosci. 2004;24(40):8720-8725.

69. Iyirhiaro GO, Zhang Y, Estey C, et al. Regulation of ischemic neuronal death by E2F4-p130 protein complexes. J Biol Chem. 2014; 289(26):18202-18213.

70. Schwab R, Bussolari R, Corvetta D, et al. Isolation and functional assessment of common, polymorphic variants of the B-MYB proto-oncogene associated with a reduced cancer risk. Oncogene. 2008;27(20):2929-2933.

71. Aleskandarany MA, Soria D, Green AR, et al. Markers of progression in early-stage invasive breast cancer: a predictive immunohistochemical panel algorithm for distant recurrence risk stratification. Breast Cancer Res Treat. 2015;151(2):325-333.

72. Lyng MB, Lænkholm AV, Tan Q, et al. Gene expression signatures that predict outcome of tamoxifen-treated estrogen receptor-positive, high-risk, primary breast cancer patients: a DBCG study. PLoS One. 2013;8(1):e54078.

73. Kerr DA, Wittliff JL. A five-gene model predicts clinical outcome in $\mathrm{ER}+/ \mathrm{PR}+$, early-stage breast cancers treated with adjuvant tamoxifen. Horm Cancer. 2011;2(5):261-271.
74. Abdel-Fatah TM, Powe DG, Ball G, et al. Proposal for a modified grading system based on mitotic index and $\mathrm{Bcl} 2$ provides objective determination of clinical outcome for patients with breast cancer. J Pathol. 2010;222(4):388-399.

75. Dawson SJ, Makretsov N, Blows FM, et al. BCL2 in breast cancer: a favourable prognostic marker across molecular subtypes and independent of adjuvant therapy received. Br J Cancer. 2010;103(5):668-675.

76. Bremer TM, Jacquemier J, Charafe-Jauffret E, Viens P, Birnbaum D, Linke SP. Prognostic marker profile to assess risk in stage I-III hormone receptor-positive breast cancer patients. Int J Cancer. 2009; 124(4):896-904.

77. Callagy GM, Webber MJ, Pharoah PD, Caldas C. Meta-analysis confirms BCL2 is an independent prognostic marker in breast cancer. BMC Cancer. 2008;8:153.

78. Linke SP, Bremer TM, Herold CD, Sauter, G, Diamond, C. A multimarker model to predict outcome in tamoxifen-treated breast cancer patients. Clin Cancer Res. 2006;12(4):1175-1183.

79. Batchelder AJ, Gordon-Weeks AN, Walker RA. Altered expression of anti-apoptotic proteins in non-involved tissue from cancer-containing breasts. Breast Cancer Res Treat. 2009;114(1):63-69.

80. Sloan CD, Shen L, West JD, et al. Genetic pathway-based hierarchical clustering analysis of older adults with cognitive complaints and amnestic mild cognitive impairment using clinical and neuroimaging phenotypes. Am J Med Genet B Neuropsychiatr Genet. 2010;153B(5):1060-1069.

81. Hoh NZ, Wagner AK, Alexander SA, et al. BCL2 genotypes: functional and neurobehavioral outcomes after severe traumatic brain injury. J Neurotrauma. 2010;27(8):1413-1427.

82. Yu KD, Di GH, Fan L, et al. A functional polymorphism in the promoter region of GSTM1 implies a complex role for GSTM1 in breast cancer. FASEB J. 2009;23(7):2274-2287.

83. Roodi N, Dupont WD, Moore JH, Parl FF. Association of homozygous wild-type glutathione S-transferase M1 genotype with increased breast cancer risk. Cancer Res. 2004;64(4):1233-1236.

84. Reed DJ. Glutathione: toxicological implications. Annu Rev Pharmacol Toxicol. 1990;30:603-631.

85. Hager MH, Morley S, Bielenberg DR, et al. DIAPH3 governs the cellular transition to the amoeboid tumour phenotype. EMBO Mol Med. 2012;4(8):743-760.

86. Vorstman JA, van Daalen E, Jalali GR, et al. A double hit implicates DIAPH3 as an autism risk gene. Mol Psychiatry. 2011;16(4): 442-451.

87. Horn D, Zhou W, Trevisson E, et al. The conserved mitochondrial twin $\mathrm{Cx} 9 \mathrm{C}$ protein $\mathrm{Cmc} 2$ is a $\mathrm{Cmc} 1$ homologue essential for cytochrome $\mathrm{C}$ oxidase biogenesis. J Biol Chem. 2010;285(20):15088-15099.
Breast Cancer - Targets and Therapy

\section{Publish your work in this journal}

Breast Cancer - Targets and Therapy is an international, peerreviewed open access journal focusing on breast cancer research, identification of therapeutic targets and the optimal use of preventative and integrated treatment interventions to achieve improved outcomes, enhanced survival and quality of life for the cancer patient.

\section{Dovepress}

The manuscript management system is completely online and includes a very quick and fair peer-review system, which is all easy to use. Visit http://www.dovepress.com/testimonials.php to read real quotes from published authors. 